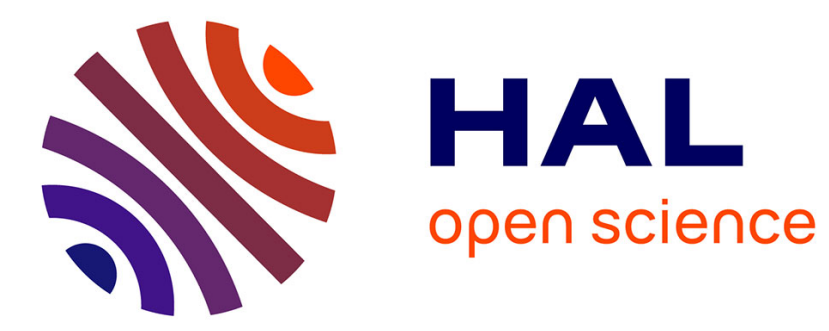

\title{
A selective review on Mumford-Shah minimizers
}

Antoine Lemenant

\section{To cite this version:}

Antoine Lemenant. A selective review on Mumford-Shah minimizers. Bollettino dell'Unione Matematica Italiana, 2016, 9, pp.69 - 113. 10.1007/s40574-016-0056-2 . hal-01468371

\section{HAL Id: hal-01468371 \\ https://hal.science/hal-01468371}

Submitted on 15 Feb 2017

HAL is a multi-disciplinary open access archive for the deposit and dissemination of scientific research documents, whether they are published or not. The documents may come from teaching and research institutions in France or abroad, or from public or private research centers.
L'archive ouverte pluridisciplinaire HAL, est destinée au dépôt et à la diffusion de documents scientifiques de niveau recherche, publiés ou non, émanant des établissements d'enseignement et de recherche français ou étrangers, des laboratoires publics ou privés. 


\title{
A selective review on Mumford-Shah minimizers
}

\author{
Antoine Lemenant
}

January 13, 2016

\section{Contents}

1 Introduction 2

2 Presentation of the functional 3

2.1 The original problem . . . . . . . . . . . . . . . . 3

2.2 Existence of minimizers . . . . . . . . . . . . . . . . 5

2.3 Almost minimizers . . . . . . . . . . . . . . . . . . 6

2.4 Blow-up limits of minimizers . . . . . . . . . . . . . . . . . . . 8

2.5 Examples of minimizers . . . . . . . . . . . . . . . 11

3 Regularity Theory 12

3.1 Euler Equation . . . . . . . . . . . . . . . . . . . . 13

3.2 Below $C^{1} \ldots \ldots \ldots \ldots \ldots \ldots$

3.3 List of $C^{1}$ regularity results . . . . . . . . . . . . . . . . . . . 15

3.4 Almost minimal sets in $\mathbb{R}^{3}$ and Jean Taylor's Theorem . . . . . . . . 23

3.5 Sketch of proof for the $C^{1}$ regularity in $\mathbb{R}^{3} \ldots \ldots$. . . . . . . . . . 25

3.6 The David-Léger paper: a little closer to the conjecture . . . . . . . . 31

3.7 The nice formula of Léger and consequences . . . . . . . . . . . . . . 31

3.8 Dimension of the Singular set and higher integrability of the gradient 32

4 Global minimizers in dimension $3 \quad 36$

4.1 If $K$ is a cone then $u$ is $1 / 2$-homogeneous . . . . . . . . . . . . . . . 36

4.2 A monotonicity formula in higher dimensions . . . . . . . . . . . . . . 38

4.3 A rigidity result for $K$ contained in a half-plane . . . . . . . . . . . . 42

5 Some links with classical mechanics $\quad 43$

5.1 The crack model of Francfort and Marigo . . . . . . . . . . . . . . . . 43

5.2 A compliance problem ..................... 52 


\section{Introduction}

The minimization of the Mumford-Shah functional was extremely studied for over 35 years in more than a hundred papers. There already exists a quite long and extensive literature on the subject, especially very well documented and thorough books [AFP00, Dav05b, MS95], and some nice review papers [Mor97, Fus03, Dav05a, Foc]. The concatenation of all the Mumford-Shah papers and books would probably be more than 2000 pages of results and proofs. Nevertheless, the main conjecture of Mumford and Shah is currently still an open and challenging problem.

In this survey paper we will obviously focus on a few aspects of the problem only, depending on our taste but also on our knowledge. We indeed chose to focus on 3 main points:

1. Dimension 2: give a picture of what is known and try to say why the conjecture is currently still open.

2. Dimension 3: expose some recent results and address some natural questions specific to that dimension.

3. Briefely explain some connections with various models from classical mechanics, especially with fracture theory and further related open questions.

In particular, we will take the opportunity to present some recent works containing regularity results in dimension 3 [Lem11, Lem09, Lem14], and we will also mention other very recent works [DLF13, DPF14, BL14] which show that the Mumford-Shah problem is still alive.

At the end we will present two recent works about fracture theory [CL13, BCL15]. Indeed, the interest in the Mumford-Shah functional is not only coming from the challenging 2D-conjecture. The same functional or some variants naturally arises in many physical models from classical mechanics that require an energy balance between a surface and a bulk term. We will try to describe at least two examples of such problems: the crack propagation model of Francfort and Marigo, and another problem involving the compliance energy. We do not wish to be exhaustive because such subject would need a review itself. Our aim will be trying to show some examples of how the tools developed for the classical Mumford-Shah problem can eventually help on those other variants coming from classical mechanics, as well as seeing how simple variants of the functional can bring highly non trivial open questions that would be of interest in regards to understand the underlying mechanical models.

A list of 12 open problems is stated all along the paper. Be aware that most of them are well known as being probably as difficult as the Mumford-Shah conjecture itself. 
Acknowledments : I wish to thank Francesco Maggi who had motivated me to write those notes, and also Guy David for reading a first version and producing a quite long list of remarks in only a few days long, but not as long as the one produced by the anonymous referee to whom I also would like to express my warm thanks. This work was partially supported by the project ANR-12-BS01-0014-01 GEOMETRYA financed by the French Agence Nationale de la Recherche (ANR). The authors acknowledge the support of the project MACRO (Modèles d'Approximation Continue de Réseaux Optimaux), funded by the Programme Gaspard Monge pour l'Optimisation of EDF and the Fondation Mathématiques Jacques Hadamard.

\section{Presentation of the functional}

\subsection{The original problem}

The Mumford-Shah functional was originally cast in dimension 2 in order to solve an image segmentation problem. In the simplest setting a given image is a $L^{\infty}$ function $g: \Omega \rightarrow \mathbb{R}$ defined on a bounded planar domain $\Omega \subset \mathbb{R}^{2}$, and one wants to find a 1-dimensional set representing its significative jump points, where we expect the edges of the image to lie. To do so, in 1989 Mumford and Shah [MS89] proposed to minimize the following functional

$$
J(u, K)=\int_{\Omega}|u-g|^{2} d x+\int_{\Omega \backslash K}|\nabla u|^{2} d x+\mathcal{H}^{1}(K)
$$

among all pairs $(u, K) \in \mathcal{A}(\Omega)$ where

$$
\mathcal{A}(\Omega)=\left\{(u, K) ; K \subset \Omega \text { is closed and } u \in W^{1,2}(\Omega \backslash K)\right\} .
$$

Actually, we could even minimize on $C^{1}(\Omega \backslash K)$ with no changes because minimizers on $W^{1,2}$ will automatically be $C^{1}$ in $\Omega \backslash K$. It would also be convenient to put some multiplicative constants to each terms in order to advantage one term with respect to another but we do not do so here because it changes nothing for the regularity theory.

Actually, minimizing the functional produces two objects : the set $K$ which represents the edges of the image (usually called the "singular set"), and at the same time a function $u$ which is smooth outside this set $K$, and that is very close to the original image in the $L^{2}$ norm. The first term of the functional is here to guarantee the latter fact. This term can be considered as a "Dirichlet condition", say, and does not count much in view of the regularity theory. The main terms of the functional are the second and third terms, which work together as two competitive terms: if for instance, the image $g$ has a sharp significant jump somewhere, in other words if there is an edge in the image, then in the minimizing process a piece of set $K$ would 
be quite useful to be added in order to save some gradient of $u$. But the price to pay is comparable to the length of the added set.

The functional works pretty well in practice. A numerical method can be obtained using the phase-field approximation of Ambrosio-Tortorelli [AT92, Bou99, BC94] or even by a direct finite elements method [CDM99, BC00].
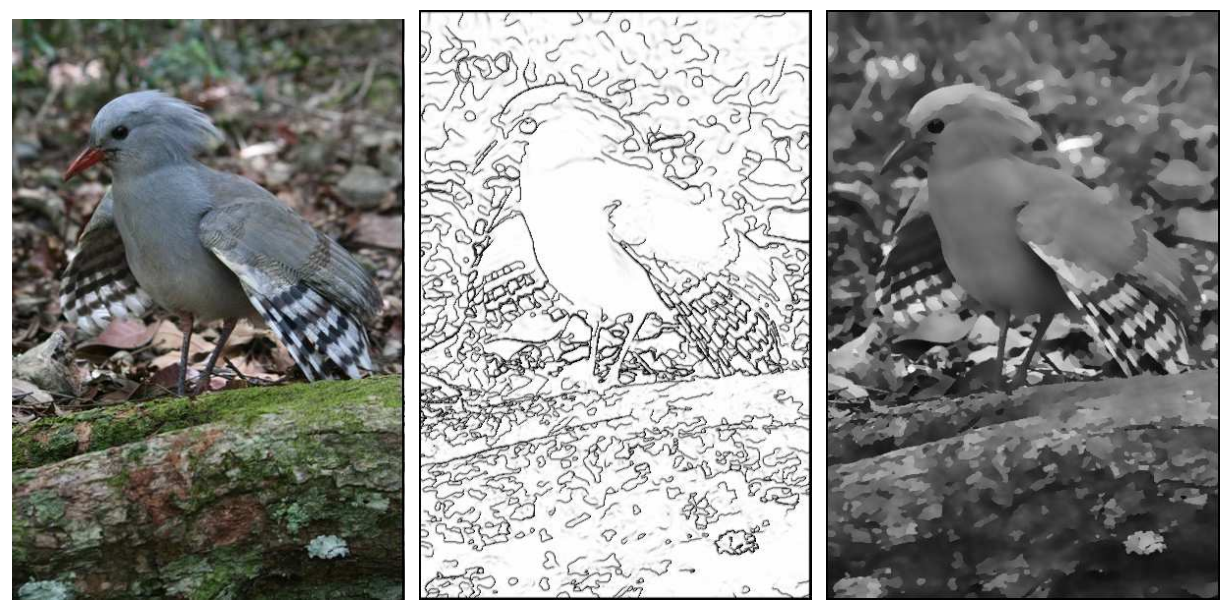

Segmentation of a Cagou by use of the Mumford-Shah functional.

Remark 2.1 (Reduced minimizers). When $(u, K) \in \mathcal{A}(\Omega)$ is a Mumford-Shah minimizer, then we could add to $K$ any piece of set of zero $\mathcal{H}^{N-1}$ measure and keep the same function $u$, without changing the fact of being a minimizer. This is why it is always important, if one wants to prove some regularity results, to assume that the pair $(u, K) \in \mathcal{A}(\Omega)$ is reduced. By reduced, we mean that one cannot remove from $K$ a piece of set without being able to extend the associated function $u$ as a $W^{1,2}$ function. From a given pair $(u, K) \in \mathcal{A}(\Omega)$, it is always possible to find an equivalent reduced pair $(\tilde{u}, \tilde{K}) \in \mathcal{A}(\Omega)$ with $\tilde{K} \subset K$ and $\mathcal{H}^{N-1}(K \backslash \tilde{K})=0$. In [Dav05b] there is even a debate between 3 different strategies to find this reduced pair. We refer to [Dav05b, Section 8] for more information about this technical but really nice question which is already very well developed in [Dav05b].

In the same paper [MS89], Mumford and Shah conjecture the following.

Open problem 2.2 (Mumford and Shah conjecture 1989). Let $(u, K)$ be a reduced minimizer of the functional $J$. Then $K$ must be a finite union of $C^{1, \alpha}$ arcs.

The conjecture is still open even though Bonnet has almost proved it in 1996 [Bon96]. We will try to give some more detail in Section 3.3.1. The $C^{1, \alpha}$ regularity here is not sharp, but it is just a first step to prove further regularity by standard elliptic theory (see Section 3.1). Up to now, what is still really missing is the finite number of curves. 


\section{$2.2 \quad$ Existence of minimizers}

The existence of minimizers was first proved by De Giorgi, Carriero, and Leaci in [DGCL89]. Their strategy is to relax the functional on $S B V$, i.e. to consider

$$
\tilde{J}(u)=\int_{\Omega}|u-g|^{2} d x+\int_{\Omega}|\nabla u|^{2} d x+\mathcal{H}^{1}\left(S_{u}\right), \quad u \in S B V(\bar{\Omega}),
$$

where $S_{u}$ is the singular set of $u$ and $\nabla u$ is the approximate gradient of $u$ (we refer to [AFP00] for the definition of $S B V$ ). It is quite easy to show that $\tilde{J}$ admits some minimizers due to the compactness result of Ambrosio [Amb89]. It is also not difficult to see that if $(u, K) \in \mathcal{A}$ is such that $\mathcal{H}^{1}(K)<+\infty$, then $u \in S B V(\Omega)$ (using [AFP00, Proposition 4.4]), and $J(u, K) \geq \tilde{J}(u)$ thus

$$
\inf _{(u, K) \in \mathcal{A}} J(u, K) \geq \min _{u \in S B V(\Omega)} \tilde{J}(u) .
$$

The issue in [DGCL89] is then to prove the reverse inequality, and this is obtained by showing that for any minimizer $u \in S B V(\Omega)$ of $\tilde{J}$, the singular set $S_{u}$ is essentially closed, namely that $\mathcal{H}^{1}\left(\overline{S_{u}} \backslash S_{u}\right)=0$. Indeed, then $\left(u, \overline{S_{u}}\right) \in \mathcal{A}$ and therefore

$$
\inf _{(w, K) \in \mathcal{A}} J(w, K) \leq J\left(u, \overline{S_{u}}\right)=\tilde{J}(u)=\min _{v \in S B V} \tilde{J}(v) .
$$

The proof of that fact is known as one of the most beautiful argument about the Mumford-Shah problem. It holds for any dimension but let us describe the main steps in dimension 2. One of the key tools is that $S_{u}$ is always contained in the complement of the set $\Omega_{0}$ of points $x \in \Omega$ for which

$$
\lim _{r \rightarrow 0} \frac{1}{r}\left(\int_{B_{r}(x)}|\nabla u|^{2} d x+\mathcal{H}^{1}\left(S_{u} \cap B_{r}(x)\right)\right)=0 .
$$

This follows from a now famous Poincaré type estimate on $S B V$ functions (one can control a truncation of $u$ minus a median by the integral of the gradient of $u$, provided the jump set is small enough).

The second ingredient is a compactness argument which provides the existence of $\varepsilon_{0}>0$ for which

$$
\frac{1}{r}\left(\int_{B_{r}(x)}|\nabla u|^{2} d x+\mathcal{H}^{1}\left(S_{u} \cap B_{r}(x)\right)\right) \leq \varepsilon_{0} \quad \Longrightarrow
$$

While the first fact holds for any $S B V$ function, the second one needs $u$ to be a minimizer. From the above two facts it can be proved that $\Omega_{0}$ must be open, and that $\overline{S_{u}}=\Omega \backslash \Omega_{0}$. Then a standard argument from measure theory says that $(2.2)$ must hold $\mathcal{H}^{1}$-a.e. on $\Omega \backslash S_{u}$ [DGCL89, Lemma 2.6] thus in conclusion we have that $\mathcal{H}^{1}\left(\overline{S_{u}} \backslash S_{u}\right)=0$. 
Let us mention that an alternative proof of existence of minimizers without using the theory of $S B V$ functions is proposed in [DMMS92] (see also [Dav05b, Section $36])$.

Finally, two other different and very recent proofs are proposed in [BL14] in any dimension and in [LF13] in dimension 2 (see also Section 3.2.1).

\subsection{Almost minimizers}

After [DGCL89], people knew that they could work equivalently on $S B V$ with the functional $\tilde{J}$ or on pairs of set and functions in $\mathcal{A}$ with the functional $J$. Then it is just a matter of education or convenience to chose which they prefer. In this paper we will follow the approach of David, Bonnet (and others) and work with $J(u, K)$ where $(u, K) \in \mathcal{A}(\Omega)$.

Also, as usual in regularity theory, one wants to work on a slightly more general definition of local almost minimizer because it includes a wider class of minimizers that are not necessarily exactly coming from the original functional $J$. We now present the definition following [Dav05b].

First let us change a little bit the definition of $\mathcal{A}(\Omega)$ in order to include the case of unbounded domains. Namely, from now on we will pose ${ }^{1}$ :

$$
\mathcal{A}(\Omega)=\left\{(u, K) ; K \subset \Omega \text { is closed and } u \in \bigcap_{R>0} W^{1,2}\left(\Omega \cap B_{R}(0) \backslash K\right)\right\} .
$$

Notice that when $\Omega$ is bounded it coincides with the preceding definition. We now give the definition of admissible competitors for the minimization property.

Definition 2.3. Let $\Omega \subset \mathbb{R}^{N},(u, K) \in \mathcal{A}(\Omega)$ and $B$ a ball such that $B \subset \Omega$. $A$ competitor for the pair $(u, K)$ in the ball $B$, is a pair $(v, L) \in \mathcal{A}$ such that:

i) $u=v$ and $K=L$ in $\Omega \backslash B$

and that moreover satisfies the following topological condition :

ii) any pair of points $x, y$ in $\Omega \backslash(B \cup K)$ which are separated by $K$ are still separated by $L$.

By "separated by $K$ " we mean that $x$ and $y$ lie in different connected components of $\Omega \backslash K$.

\footnotetext{
${ }^{1}$ actually, a posteriori we could be less exigent on $u$ because being a minimizer automatically implies that $u$ belongs to all the $W^{1,2}\left(\Omega \cap B_{R}(0) \backslash K\right)$ but we prefer to not proceed like this to avoid some possible confusion.
} 


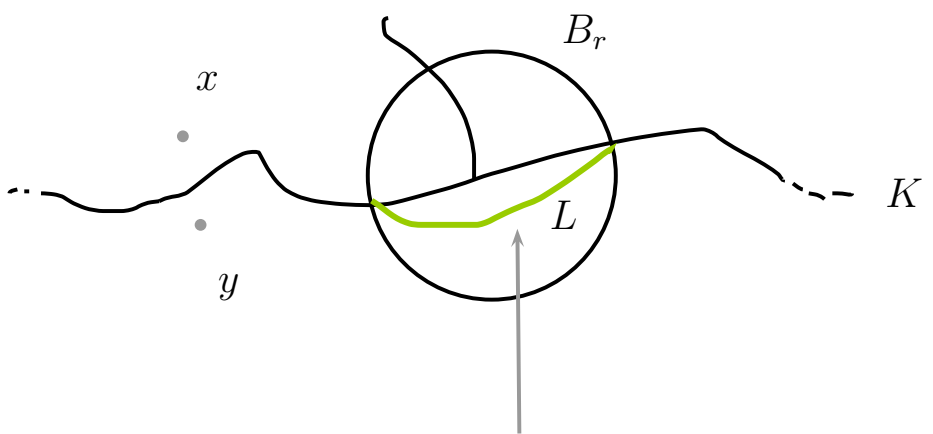

A competitor in $B_{r}$ must preserve the separation between $x$ and $y$ outside the ball.

Why assuming this topological condition ? First of all, notice that this condition is very natural, at least for a moral reason: it says that one cannot win too easily some length in a ball $B$ by just erasing it creating a big hole in $K$. For instance, we claim that without assuming this topological condition in the definition, a line in $\mathbb{R}^{2}$ with $u$ equal to two constants on each side would not be a Mumford-Shah minimizer in the plane. Let us check this fact: let $u$ be locally constant in $\mathbb{R}^{2} \backslash K$ with $K$ being the horizontal line in the plane passing through the origin. We then consider the (non topological) competitor $(v, L)$ in the ball $B_{2 R}(0)$ with $L=K \backslash B_{R}$ and $v=u \varphi$, where $\varphi$ is a radial Lipschitz cut-off function equal to 1 outside $B_{2 R}$, equal to 0 in $B_{R}$, and satisfying $|\nabla v| \leq C \frac{1}{R}$. By minimality of $(u, K)$ we would have

$$
0+4 R \leq C\left|B_{2 R} \backslash B_{R}\right| \frac{1}{R^{2}}+2 R \Rightarrow 2 R \leq C,
$$

and we get a contradiction by letting $R \rightarrow+\infty$.

But of course we expect a line being a minimizer (it is indeed a topological one), because it is a possible blow-up limit at a regular point of a minimizer. Historically, this condition was found by Bonnet while he took blow-up limits of minimizers. Due to some compactness issues in the blow-up procedure, the limiting object inherits this topological condition. Then David decided to study almost minimizers with this condition, that he called topological almost minimizers (or TRLAM for topological reduced local almost minimizers). The reason was principally for sake of unification, i.e. give a definition that includes both minimizers of the functional (local ones) and at the same time global minimizers, which are blow-up limits of minimizers (the definition will be given just below). Anyway this is not a serious restriction because in practice it will always be easy to satisfy this topological condition while constructing competitors, which is the main issue to obtain any kind of result on minimizers. For the record, David also uses the same topological condition in his class of almost minimizing sets, that he still call MS-minimizers (MS for MumfordShah) but where no function $u$ exists anymore [Dav09, Dav10] (we will see this in Section 3.4).

We now define our class of minimizers. 
Definition 2.4 (Almost minimizer). Let $\Omega \subset \mathbb{R}^{N} . A$ pair $(u, K) \in \mathcal{A}(\Omega)$ is an almost Mumford-Shah minimizer, or in short, a minimizer, if there exists a nondecreasing function $h: \mathbb{R}^{+} \rightarrow \mathbb{R}^{+}$with $\lim _{r \rightarrow 0} h(r)=0$ such that, for all $B_{r} \subset \Omega$, and for all competitor $(v, L)$ in $B$ we have

$$
\int_{B \backslash K}|\nabla u|^{2} d x+\mathcal{H}^{N-1}(K \cap B) \leq \int_{B \backslash L}|\nabla v|^{2} d x+\mathcal{H}^{N-1}(L \cap B)+r^{N-1} h(r) .
$$

The function $h$ will be called the gauge associated to $(u, K)$. In general, the regularity theory will say that when $h(r)=C r^{\alpha}$, then any almost minimizer will be of class at least $C^{1, \alpha^{\prime}}$ almost everywhere, for some $0<\alpha^{\prime}<\alpha$.

It is not difficult to see that any minimizer of the functional $J$ defined earlier, is in particular an almost minimizer with $h(r)=C_{N}\|g\|_{\infty}^{2} r$.

Another class of minimizers playing an important role in the regularity theory is the following notion of global minimizers, coming as blow-up limits of almost minimizers. They are also called Bonnet-minimizers or B-minimizers in [AFP00]. The classification of all global minimizers in dimension 2 would be a way to solve the Mumford-Shah conjecture (Open Problem 2.2). It is also a nice open problem to find some in dimension 3, other than the classical ones (see Section 4).

Definition 2.5 (Global minimizer). A pair $(u, K) \in \mathcal{A}\left(\mathbb{R}^{N}\right)$ is a global MumfordShah minimizer (or in short, global minimizer), if for any ball $B \subset \mathbb{R}^{N}$, and for any competitor $(v, L)$ for $(u, K)$ in $B$ we have

$$
\int_{B \backslash K}|\nabla u|^{2} d x+\mathcal{H}^{N-1}(K \cap B) \leq \int_{B \backslash L}|\nabla v|^{2} d x+\mathcal{H}^{N-1}(L \cap B) .
$$

We finish this section with two important remarks.

Remark 2.6 (Terminology employed in this paper). In all the sequel, a MumfordShah minimizer will most of the time refer to an almost minimizer in the sense of Definition 2.4, which includes in particular the possibility of being a global minimizer, or a local minimizer of the functional. Sometimes we will even say, in short, "minimizer". A few times later, as it would come clear from the discourse, it would only concern minimizers of the functional.

\subsection{Blow-up limits of minimizers}

One of the key ingredient in the regularity result of Bonnet, is taking blow-up and blow-in limits of minimizers. More precisely, let $(u, K) \in \mathcal{A}(\Omega)$ be a minimizer with gauge function $h(t)$. For $r>0$ and for any $x_{0} \in \Omega$ let $\left(u_{r}, K_{r}\right)$ be the new minimizer 
in $\frac{1}{r}\left(\Omega-x_{0}\right)$ associated to the gauge function $h(r t)$ defined by ${ }^{2}$

$$
u_{r}(x):=\frac{1}{\sqrt{r}} u\left(r x+x_{0}\right) \text { and } K_{r}:=\frac{1}{r}\left(K-x_{0}\right) .
$$

Extracting sequences as $r \rightarrow 0$ which converge in a fairly weak sense is not very difficult and follows from standard compactness arguments, together with the uniform concentration property (see Section 3.2.2) which guarantees the lower-semicontinuity of $\mathcal{H}^{N-1}(K)$ along a sequence of minimizers. The next difficult part is to show that this convergence holds strongly in $L_{l o c}^{2}\left(\mathbb{R}^{N}\right)$ for the gradients, and that the limiting object is still a minimizer (precisely, a global minimizer as in Definition 2.5). This was done in [Bon96] for minimizers of the Mumford-Shah functional in dimension 2 (see also [Dav05b, Section D.40] in a general framework).

The limiting pair is called a blow-up limit at $x_{0}$. If on the opposite $r \rightarrow+\infty$ it is called a blow-in limit, which is only used in particular cases, for instance when $\Omega=\mathbb{R}^{N}$ and $(u, K)$ is a global minimizer.

The key point to obtain some regularity is then to classify all the possible blow-up limits. This was done in dimension 2 in [Bon96] assuming that the singular set is connected. We will come back later to this fact (see Section 3.3.1). The classification in dimension 3 is a natural and delicate open problem that we will discuss in Section 4.

Let us now be more precise about the definition of convergence of a blow-up sequence. For all $k \in \mathbb{N}$ we denote by $B_{k}$ the ball $B_{k}(0)$. If $K$ and $K^{\prime}$ are two compact subsets of $\mathbb{R}^{N}$ the local Hausdorff distance in $B_{k}$ between $K$ and $K^{\prime}$ is

$$
d_{k}\left(K, K^{\prime}\right)=\max \left(\sup _{x \in K \cap B_{k}} \operatorname{dist}\left(x, K^{\prime}\right), \sup _{x \in K^{\prime} \cap B_{k}} \operatorname{dist}(x, K)\right) .
$$

We say that a sequence of closed sets $K_{n} \subset \mathbb{R}^{N}$ converge to a set $K \subset \mathbb{R}^{N}$ when

$$
\lim _{n \rightarrow+\infty} d_{k}\left(K_{n}, K\right) \rightarrow 0 \quad \forall k \geq 0
$$

Subsequently, the convergence of a sequence of minimizers $\left(u_{k}, K_{k}\right)$ will be understood in the following way.

Definition 2.7. We say that a sequence of couples $\left(u_{k}, K_{k}\right) \in \mathcal{A}\left(\Omega_{k}\right)$ converges to some $\left(u_{0}, K_{0}\right) \in \mathcal{A}\left(\mathbb{R}^{N}\right)$ if the following holds.

\section{1. $K_{k}$ converges to the set $K$ in the sense of (2.4).}

\footnotetext{
${ }^{2}$ Sometimes it is convenient to make the reference point $x_{0}$ actually depend on $r$ too but this is used so rarely that we decided to not talk about it here (however it is one of the key tool in the proof of the regularity result of Bonnet, for instance to be able to show that they are only a finite number of triple junctions in each isolated connected components [Bon96, page 508]).
} 
2. for any connected component $U \subseteq \mathbb{R}^{N} \backslash K_{0}$, there exists a sequence of numbers $a_{k}$ such that $u_{k}-a_{k}$ converges to $u_{0}$ strongly in $L^{1}(H)$, for every compact set $H \subset U$.

3. $\nabla u_{k} \rightarrow \nabla u_{0}$ strongly in $L^{2}\left(B_{R}\right)$, for every $R>0$.

The following statement summarizes the type of compactness result regarding to blow-up sequences that we shall need in general. It is mainly coming from gluing together several Propositions contained in the book [Dav05b].

Theorem 2.8. Let $(u, K)$ be a reduced minimizer in $\Omega$ and for some $x_{0} \in \Omega$ and for a sequence $r_{k} \rightarrow 0$ let $\left(u_{r_{k}}, K_{r_{k}}\right)$ be the blow-up sequence defined by (2.3). Then, there exists a subsequence $r_{k_{l}} \rightarrow 0$ such that $\left(u_{r_{k}}, K_{r_{k_{l}}}\right)$ converges to some $\left(u_{0}, K_{0}\right)$ in the sense of Definition 2.7. Moreover $\left(u_{0}, K_{0}\right)$ is a reduced global minimizer in $\mathbb{R}^{N}$.

Elements of proof. The convergence of $K_{r}$ and $u_{r}$, together with a weak convergence for $\nabla u_{r}$ in $L^{2}\left(B_{M}\right)$ are consequences of standard compactness results, as explained for instance in [Dav05b, Proposition D.37.8]. The starting point is the fact that $u_{r}$ have a uniform Dirichlet energy in $B_{R}$, due to the classical energy estimate for Mumford-Shah minimizers coming from taking $\left(u 1_{\Omega \backslash B_{r}},\left(K \backslash B_{r}\right) \cup \partial B_{r}\right)$ as competitor,

$$
\int_{B_{r}}|\nabla u|^{2} d x \leq C r^{N-1}
$$

This allows to get weak-compactness in $L_{l o c}^{2}$ for the gradients. The compactness for the sets $K_{r}$ comes from the classical Blaschke selection theorem.

This "weak" convergence is enough to obtain that $\left(u_{0}, K_{0}\right)$ is a reduced global minimizer. This fact is far from obvious but a proof is, for instance, given in [Dav05b, Theorem D.40.9]. In addition, by [Dav05b, Proposition D.37.18] (which is just semicontinuity with respect to the weak convergence), we get

$$
\int_{B_{R} \backslash K_{0}}\left|\nabla u_{0}\right|^{2} d x \leq \liminf _{l} \int_{B_{R} \backslash K_{r_{k_{l}}}}\left|\nabla u_{r_{k_{l}}}\right|^{2} d x
$$

Finally, the reverse inequality in the above with compact balls and a limsup is again not obvious, but follows from [Dav05b, Corollary D.38.48], which together with the weak convergence implies strong convergence in $L^{2}\left(B_{R}\right)$ for the gradients.

Remark 2.9. Actually one can show that the convergence in compact sets of $\mathbb{R}^{N} \backslash K_{0}$ is even better: it is a uniform convergence [Dav05b, Proposition D.37.25].

Remark 2.10. One can derive a similar statement for blow-in limits (i.e. $r_{k} \rightarrow+\infty$ ) in the case when $\Omega=\mathbb{R}^{N}$ and $h=0$. 


\subsection{Examples of minimizers}

We end this presenting section by giving explicit examples of minimizers, which are actually quite difficult to build in general. In [DPS99] it is shown that provided $\lambda>0$ is large enough, the minimizer of the functional (2.1) associated to $g=\lambda \mathbf{1}_{B_{R}}$ in $\Omega=B_{R^{\prime}}$ with $R^{\prime}>R$ is unique, and given by $\left(g, \partial B_{R}\right)$, as we could intuitively expect. Observe that it is not the same if we replace the euclidean ball $B_{R}$ by, for instance, a square. Indeed, a square will never minimize the functional because if would necessarily be $C^{1}$ by Bonnet's regularity result.

But the most efficient way to check if a candidate is minimizing or not is probably to use the clever calibration method that was proposed in [ABDM03]. It was used for instance to prove that the so called propeller, i.e. three half lines meeting by 3 with angles of 120 degree with associated locally constant function, with large enough jumps, is a minimizer.

Some other examples are known provided we add a parameter $\beta>0$ in front of one term of the functional, as follows:

$$
J_{\beta}(u, K)=\beta \int_{\Omega}|u-g|^{2} d x+\int_{\Omega \backslash K}|\nabla u|^{2} d x+\mathcal{H}^{1}(K) .
$$

It is straitforward to prove that, assuming $g \in S B V(\Omega)$, any sequence of minimizers $u_{\beta}$ minimizing $J_{\beta}$ converges to $g$ in $L^{2}$ when $\beta \rightarrow+\infty$. In [ABDM03] some more accurate result is proved using the calibration technique: it is showed that a solution of the Neumann problem $-\Delta u=\beta\left(u-g\right.$ ) is a minimizer (with $K=\emptyset$ ) for $J_{\beta}$ provided that $\beta$ is large enough (see also the proof of [Fus03, Theorem 3.1. (i)] for an alternative argument without calibration, due to Chambolle).

The calibration was then used later in several papers [Mor02b, Mor02a, DMMM00, Mor02c, MM01] to find other particular examples. One of those is the following nice result:

Theorem 2.11. [Mor02c] Suppose that $\Gamma$ is a closed hypersurface of class $C^{2, \alpha}$ in a smooth domain $\Omega$ and that $g$ is a Lipschitz function in $\Omega \backslash \Gamma$ jumping across $\Gamma$. Then for $\beta$ large enough, the minimizer $(u, K)$ of $J_{\beta}$ is unique and satisfies $K=\Gamma$.

Another famous result about Mumford-Shah minimizers is the extremely long paper [BD01] which contains the proof of the fact that the well-known cracktip solution defined by the pair

$$
\left.u(r, \theta)=\sqrt{\frac{2}{\pi}} r \sin (\theta / 2), r>0, \theta \in\right]-\pi, \pi\left[, \quad \text { and } \quad K=\mathbb{R}^{-} \times\{0\},\right.
$$

is a global minimizer in the plane. It would be very nice to find a way to prove the same result using the calibration method of [ABDM03] but nobody succeeded so far. It is even not known whether there exists a Mumford-Shah minimiser in $\Omega \subset \mathbb{R}^{2}$ 
whose blow-up limit at some point $x$ is the cracktip function except, of course, for the cracktip itself.

Open problem 2.12 (Asked in [ABDM03] and in Page 414 of [Dav05b]). Find a calibration for the crackip function in order to recover the result in [BD01] with a much simpler proof.

\section{Regularity Theory}

Here we try to describe some known results about regularity of minimizers. This section is divided in two main parts, corresponding to results below $C^{1}$ (Ahlforsregularity, rectifiability, etc.) and then $C^{1}$-regularity results.

We will mostly focus on the $C^{1}$ regularity results, for which at least 4 different works will be presented. They are always based upon the same strategy: control the quantity $\int_{B_{r} \backslash K}|\nabla u|^{2} d x$ and prove that, at some particularly well chosen points, it behaves like $C r^{1+\alpha}$. Indeed, if this is true, then we are morally reduced to an almost minimizing set, i.e. which almost minimizes $\mathcal{H}^{N-1}$ with an excess of minimality controlled by $\mathrm{Cr}^{1+\alpha}$, and we can then apply the regularity theory for almost minimizing sets.

But this strategy is doomed to failure just before it started: indeed, proving an estimate like $\int_{B_{r} \backslash K}|\nabla u|^{2} d x \leq C r^{1+\alpha}$ is already a sort of regularity result on the function $u$, which is normally hard to obtain without knowing any regularity on the set $K$.

This is why one needs to do both at the same time, controlling the geometry of $K$ provided the energy stays small enough, and vice versa, prove that the energy decays when some geometrical quantity on $K$ stay small, and at the end iterate both estimates and pray for them to bootstrap in the good direction. This is what we can find behind the proofs of [AFP97], [Dav96] or [Lem11] and this is why the regularity theory for Mumford-Shah gets quickly involved.

In dimension 2 only, there is an elegant way to bypass this problem that we will try to present briefly, which was found by Bonnet in [Bon96]. Namely, a nice monotonicity formula gives the estimate $\int_{B_{r}}|\nabla u|^{2} d x \leq C r^{1+\alpha}$ for free at any "flat point" (i.e. where $K$ admits a tangent line, and this occurs $\mathcal{H}^{1}$-a.e. because it is rectifiable), as soon as $K$ is a connected set. Unfortunately this argument works well only in dimension 2. One could find later in Lemma 4.6 a generalization of this monotonicity result in higher dimension, but it works only in very particular situations.

In Section 3.3 we will not present all the $C^{1}$ proofs, but only the one of Bonnet, and the main ideas of [Lem11]. 


\subsection{Euler Equation}

Here we return to the original Mumford-Shah functional and assume that $(u, K)$ is a minimizer. Assume that $K$ is the graph of a smooth function $f$ of regularity $C^{1, \alpha}$ in $B(x, r)$, and also that $g$ is sufficiently smooth. Since $u$ minimizes some energy of elliptic type, it is the weak solution of

$$
\left\{\begin{aligned}
-\Delta u+u & =g & & \text { in } B(x, r)^{ \pm} \\
\frac{\partial u}{\partial n} & =0 & & \text { on } K \cap B(x, r) \\
-\operatorname{div}\left(\frac{\nabla f}{\sqrt{1+\nabla f^{2}}}\right)^{2} & =\left[|\nabla u|^{2}+(u-g)^{2}\right]^{ \pm} & & \text {on } K \cap B(x, r) .
\end{aligned}\right.
$$

The notation $[u]^{ \pm}$used in the right-hand side of the last equation means the jump of $u$ across $K$, i.e. the difference of the traces on both sides of $K$. A consequence of that system together with a classical bootstrap argument is that whenever $K$ is $C^{1, \alpha}$ and $g$ is $C^{k, \alpha}$, then $K$ is $C^{k+2, \sigma}$ [AFP00, Theorem 7.42].

It is also known that $K$ is analytic when $g$ is, and this was proved in [KLM05] answering a question by De Giorgi.

The main issue is then to prove some preliminary $C^{1, \alpha}$ regularity.

\subsection{Below $C^{1}$}

Before that $C^{1}$ results appeared, a list of mild regularity properties have been established on Mumford-Shah minimizers. Some of them are a bit exotic and will not be stated here (like the uniform projection property [DK91, Lég94] or the bissection property [Sol97]), but some others like the Alhfors-regularity or uniform concentration property are really useful. Some of them have been originally proved for minimizers of the functional only, but most of the arguments have been reproduced in a more general framework in [Dav05b] for almost-minimizers.

\subsection{1 $K$ is locally Ahlfors-Regular.}

If $(u, K)$ is a minimizer, then we know that $K$ is Ahlfors-Regular, which means that there exists some $r_{0}>0$ and $C_{1}, C_{2}>0$ for which

$$
C_{1} \leq \frac{\mathcal{H}^{N-1}\left(K \cap B_{r}(x)\right)}{r^{N-1}} \leq C_{2}, \quad \forall x \in K, \quad \forall r \leq r_{0} \quad \text { s.t. } B(x, r) \subset \Omega .
$$

This notion is a very weak "regularity notion" which does not imply any regularity of the set in the usual sense but is still commonly referred as a "regularity result". One of the main reason that motivated people to establish such regularity property is that, proving it on minimizers of the $S B V$ version of the functional, it implies the essential closeness of the jump set thus the existence of minimizers for 
the Mumford-Shah functional on pairs $(u, K) \in \mathcal{A}(\Omega)$ (the proof follows the one described in Section 2.2 with minor modifications). But knowing it directly on minimizers $(u, K) \in \mathcal{A}(\Omega)$ has also its own importance, because it implies some uniform estimates: it is the first step to prove uniform rectifiability of the set, uniform concentration property, Carleson-measure type estimates, for instance [Dav05b, Chapter C].

Finding an upper bound is rather easy, with constant $C_{2}=\mathcal{H}^{N-1}\left(\mathbb{S}^{N-1}\right)+h\left(r_{0}\right)$. Indeed, if $(u, K)$ is a minimizer, one can take the competitor $(v, K)$ in the ball $B_{r}(x)$ defined by $v=u \mathbf{1}_{\boldsymbol{\Omega} \backslash \mathbf{B}}$ and $L=(K \backslash B) \cup \partial B$ which directly gives the estimate

$$
\int_{B}|\nabla u|^{2} d x+\mathcal{H}^{N-1}(K \cap B) \leq\left(\mathcal{H}^{N-1}\left(\mathbb{S}^{N-1}\right)+h\left(r_{0}\right)\right) r^{N-1} .
$$

The lower bound is more delicate, has a long story and several different proofs. The first estimate of that kind was proved in [CL90] using the technics introduced in [DGCL89]. In particular it follows from a compactness argument. An alternative and more direct approach called "excision technic" is given in [DMMS92] in dimension 2, that was extended in [Sol97] for higher dimensions. Later, Siaudeau [Sia03] proved it for a more general class of minimizers called "quasi-minimizers" (i.e. where the minimality is changed up to loose a multiplicative constant in front of the functional ${ }^{3}$ ). The proof relies strongly on the technics of [DGCL89] but written in a non- $S B V$ fashion (see also [Dav05b, Chapter C]). More recently in [BL14] a new approach was found related to a monotonicity formula obtained via an induction argument on the dimension. Finally, via yet a new approach in [LF13], the lower bound is given with $C_{1}=\pi / 2^{24}$ in dimension 2 , which is not supposed to be optimal but has the merit to be explicit.

\subsection{2 $K$ has the uniform concentration property.}

The uniform concentration property is a very important case of lower semisontinuity introduced by Dal Maso, Morel and Solimini [DMMS92] which allows to take limits of minimizers and prove that the limit is still a minimizer. Indeed, it is very classical that $K \mapsto \mathcal{H}^{N-1}(K)$ may not be lower semicoutinuous with respect to Hausdorff convergence in general. A very famous special case in dimension 2 is the so called Golab Theorem which says that it holds true along a sequence of compact connected sets. The uniform concentration property provides a sort of generalization which works in any dimension, and says that $K \mapsto \mathcal{H}^{N-1}(K)$ is lower semicontinuous for a uniform sequence of uniformly concentrated sets, which actually holds for a sequence of Mumford-Shah minimizers. We shall not enter into more detail here but refer the reader to [Dav05b, Section 35].

\footnotetext{
${ }^{3}$ Notice that this notion of quasi-minimality is different from the quasi-minimality of [AFP00], in which according to their terminology, a quasi-minimizer is a $S B V$ version of what we call here almost-minimizers.
} 


\subsection{3 $K$ is uniformly rectifiable.}

Uniform rectifiability is a sort of quantitative notion of rectifiability, that was historically introduced and made famous by David and Semmes [DS91] in connection with the boundeness in $L^{2}$ for a class of singular integral operators with CalderónZygmund kernel.

We shall not enter too much in detail here about the notion of uniform rectifiability. Let us just say that there exists several ways of defining uniform rectifiability, and the major part of [DS91] is indeed to prove that they are all equivalent. For a set of dimension 1 at least, there exists a simple way: an Ahlfors-Regular set $K \subset \mathbb{R}^{N}$ is 1-uniformly rectifiable if it is contained in a "regular curve", which is a Lipschitz curve which have an Alhfors-regular image. To define uniform rectifiability in higher dimension, let us introduction the beta number which measures the flatness of $K$ in $B(x, t)$,

$$
\beta_{1}(x, t)=\inf _{P} \frac{1}{t^{d}} \int_{E \cap B(x, t)} \frac{\operatorname{dist}(x, P)}{t} d y,
$$

where the infimum is taken over all $d$-planes $P$. Then, a $d$-Ahlfors regular set is uniformly rectifiable, if and only if $\mu:=\beta_{1}(x, t)^{2} \frac{d x d t}{t}$ is a Carleson measure on $E \times \mathbb{R}_{+}$. This means that $\mu(B(x, r) \times[0, R]) \leq C R^{d}$ for all $x \in K$ and $R>0$. In other words $\mu$ behaves like $R^{d}$ on the product $E \times \mathbb{R}^{+}$, thus $K$ must be "flat" very often.

One can find a very nice survey on uniformly rectifiable sets on Guy David's webpage (find the file called "Notes-Parkcity.pdf" in the preprint page).

In [DS96b, DS96a] David and Semmes proved that the singular set of a MumfordShah minimizer is uniformly rectifiable. This result is interesting for at least two reasons. Firstly it contains or implies a series of previous known results like, rectifiability, uniform projection property, uniform concentration property, Ahlfors regularity, etc. Secondly, it provides some quantitative estimates on the flatness of $K$, saying that $K$ is locally very close to a hyperplane in many balls, at any scale with radius controlled from below. This fact can be exploited to derive some estimates on the Hausdorff dimension of the singular set, as was first used by David in [Dav96] and then by Rigot [Rig00] (see also Section 3.8). It is also related to a notion of "porosity" that was used in [DPF14] to prove higher integrability of the gradient (see Section 3.8).

\subsection{List of $C^{1}$ regularity results}

Here we present the main $C^{1}$-regularity results. Three of them appeared almost the same year 1996 [Dav96], [Bon96], [AFP97] and a last one specific to dimension 3 was done later in [Lem08, Lem11]. All of them have in common to contain a proof 
for $C^{1}$-almost everywhere regularity, but each of them has its own interest that we try to describe below.

\subsection{1 $C^{1}$-Result of Bonnet}

The closer result to the Mumford-Shah conjecture is probably the following one obtained by Bonnet [Bon96]. The key ingredient is a monotonicity formula for the Dirichlet integral which permitted him to classify the blow-up limits. This work is purely 2-dimensional.

Theorem 3.1. [Bon96] Let $(u, K)$ be a reduced minimizer. Then the Mumford-Shah conjecture is true for every isolated connected component of $K$. Precisely, if $G$ is an isolated connected component of $K$, then it is the union of a finite set of $C^{1}$ arcs, $C^{1,1}$ away from crack-tips and that can only merge through triple junctions.

The proof of Bonnet relies on a very nice monotonicity formula which allows him to classify the possible blow-up limits. Let us sketch the proof here.

Proposition 3.2 (Monotonicity Formula of Bonnet). Let $\Omega \subset \mathbb{R}^{2}$ be open, and assume that $K \subset \Omega$ is a closed and connected set of finite length. Let $u$ be an energy minimizer i.e. satisfying

$$
\int_{B \backslash K}|\nabla u|^{2} d x \leq \int_{B \backslash K}|\nabla v|^{2} d x
$$

for any $B \subset \Omega$ and for any $v$ that is equal to $u$ in $\Omega \backslash B$ (the function $u$ is therefore the weak solution of a Neumann problem, $\Delta u=0$ in $\Omega \backslash K$ and $\frac{\partial u}{\partial \nu}=0$ on $\left.K\right)$.

For any point $x_{0} \in K$ we denote

$$
E(r):=\int_{B\left(x_{0}, r\right) \backslash K}|\nabla u|^{2} d x
$$

Then $r \mapsto E(r) / r$ is an increasing function of $r$ on $\left(0, \operatorname{dist}\left(x_{0}, \partial \Omega\right)\right)$. As a consequence, the limit $\lim _{r \rightarrow 0}(E(r) / r)$ exists and is finite.

Moreover, if $r \mapsto E(r) / r$ is a non zero constant on some interval $(a, b)$, then for $r \in(a, b), K \cap \partial B_{r}$ is a single point and the restriction of $u$ on $\partial B_{r} \backslash K$ for $r \in(a, b)$ must be the optimal function in Wirtinger's inequality.

Proof. Let us assume without loss of generality that $x_{0}$ is the origin. Firstly, it is easy to show that $E$ admits a derivative a.e. and

$$
E^{\prime}(r):=\int_{\partial B(0, r) \backslash K}|\nabla u|^{2} d x .
$$


In addition $E$ is absolutely continuous. Therefore, to prove the monotonicity of $r \mapsto \frac{1}{r} E(r)$, it is enough to prove the inequality

$$
E(r) \leq r E^{\prime}(r) \quad \text { for a.e. } r \leq r_{0},
$$

because this implies $\left(\frac{1}{r} E(r)\right)^{\prime} \geq 0$ a.e.

We will need Wirtinger's inequality (see e.g. page 301 of [Dav05b]), i.e. for any arc of circle $I_{r} \subset \partial B(0, r)$ and for $g \in W^{1,2}\left(I_{r}\right)$ we have

$$
\int_{I_{r}}\left|g-m_{g}\right|^{2} d \sigma \leq 4\left(\frac{\left|I_{r}\right|}{2 \pi}\right)^{2} \int_{I_{r}}\left|g^{\prime}\right|^{2} d \sigma
$$

where $m_{g}$ is the average of $g$ on $I_{r}$. The constant 4 here is optimal, and is achieved by the function $\sin (\theta / 2)$ on the arc of circle $\{\theta \in]-\pi, \pi[\}$. This will be needed later.

Observe that since $K$ has a finite length, we know that $\sharp K \cap \partial B(0, r)$ is finite for a.e. $r \in\left(0, r_{0}\right)$. We take such a radius and decompose $S_{r}:=\partial B(0, r) \backslash K$ into a finite number of arcs of circle denoted $I_{j}$, for $j=1 . . N$. Moreover since $K$ is closed and connected, for each $j$ there exists a geodesic simple curve $F_{j} \subset K$ connecting the two endpoints of $I_{j}$ (here is where connectedness plays a role). We denote by $D_{j}$ the domain delimited by $I_{j}$ and $F_{j}$. Observe that the domains $D_{j}$ for $j=1 . . N$ are disjoint.

The Gauss-Green formula (that can by justified easily here by a variational argument) applied in $B(0, r)$ yields

$$
\int_{B(0, r) \backslash K}|\nabla u|^{2} d x=\sum_{i=1}^{N} \int_{I_{j}} u \frac{\partial u}{\partial \nu} d \sigma,
$$

and applied in $D_{j}$ gives

$$
\int_{I_{j}} \frac{\partial u}{\partial \nu} d x=\int_{D_{j}} \Delta u d x=0 .
$$

Denoting by $m_{j}$ the average of $u$ on $I_{j}$ we deduce that

$$
\int_{I_{j}} u \frac{\partial u}{\partial \nu} d \sigma=\int_{I_{j}}\left(u-m_{j}\right) \frac{\partial u}{\partial \nu} d \sigma .
$$

Then by use of Cauchy-Schwarz inequality and $a b \leq \frac{1}{2 \lambda} a^{2}+\frac{\lambda}{2} b^{2}$ we can write

$$
\begin{aligned}
\int_{I_{j}}\left|u-m_{j}\right|\left|\frac{\partial u}{\partial \nu}\right| d \sigma & \leq\left(\int_{I_{j}}\left|u-m_{j}\right|^{2}\right)^{\frac{1}{2}}\left(\int_{I_{j}}\left|\frac{\partial u}{\partial \nu}\right|^{2}\right)^{\frac{1}{2}} \\
& \leq \frac{1}{2 \lambda} \int_{I_{j}}\left|u-m_{j}\right|^{2}+\frac{\lambda}{2} \int_{I_{j}}\left|\frac{\partial u}{\partial \nu}\right|^{2} .
\end{aligned}
$$


Using Wirtinger inequality and setting $\lambda=2 r$ we deduce that

$$
\begin{aligned}
\int_{I_{j}}\left|u-m_{j}\right|\left|\frac{\partial u}{\partial r}\right| d \sigma & \leq \frac{4 r^{2}}{2 \lambda} \int_{I_{j}}\left|u_{\tau}\right|^{2}+\frac{\lambda}{2} \int_{I_{j}}\left|\frac{\partial u}{\partial \nu}\right|^{2} \\
& \leq r \int_{I_{j}}\left|u_{\tau}\right|^{2}+r \int_{I_{j}}\left|\frac{\partial u}{\partial \nu}\right|^{2} \\
& =r \int_{I_{j}}|\nabla u|^{2} .
\end{aligned}
$$

Finally summing over $j$, we get (3.2) and the monotonicity is proved.

The last conclusion of the proposition then follows from the case of equality in the above inequalities.

Theorem 3.3 (Classification of connected global minimizers in $\left.\mathbb{R}^{2}\right)$. Let $(u, K)$ be a global minimizer in $\mathbb{R}^{2}$ such that $K$ is connected. Then it belongs to the following list.

1. $K=\emptyset$ and $u$ is constant.

2. (Line) $K$ is a line and $u$ is constant on each side.

3. (Propeller) $K$ is the union of three half-lines meeting at their tip by angles of 120 degree.

4. (Cracktip) Up to translation, rotation, or additional constant, $K$ is a half line and $u$ is equal to the cracktip function defined in (2.6).

Elements of proof. Let $(u, K)$ be a global minimizer, and assume that $K$ is connected. The key ingredient is the monotonicity formula. It says that the quantity $\varphi(r)=E(r) / r$ is nondecreasing (Proposition 3.2). Thus, let $\varphi(+\infty)$ and $\varphi(0)$ be the respective limits for $r$ going to 0 and $+\infty$. First we notice that $\varphi(+\infty)$ is finite, due to the estimate $\int_{B_{r}}|\nabla u|^{2} d x \leq 2 \pi r$ valid for any global minimizer. Next, we use the blow-up and blow-in procedure, to obtain at the limit two new pairs $\left(u_{0}, K_{0}\right)$ and $\left(u_{\infty}, K_{\infty}\right)$ which are again global minimizers with connected singular sets, for which their respective quantity $\varphi(r)$ is constantly equal to $\varphi(\infty)$ for the blow-in and $\varphi(0)$ for the blow-up. By the last conclusion of the monotonicity result (Proposition 3.2), it follows that $\varphi(\infty)$ and $\varphi(0)$ can be only equal to 0 or 1 . Indeed, in the case when it is not 0, using the last conclusion of Proposition 3.2 we deduce that $u$ is of the form $C(r)+\alpha(r) \sin \left(\frac{\theta-\theta(r)}{2}\right)$ for some $C(r), \alpha(r)$ and $\theta(r)$. But since $u$ is harmonic it follows that $\theta(r)=\theta_{0}, \alpha(r)=\alpha \sqrt{r}$ and $C(r)=C$. Finally, the constant $\alpha$ must be equal to $\sqrt{2 / \pi}$ due to the fact that we have a Mumford-Shah minimizer, which implies some variational equalities (see [Dav05b, Page 406]), leading to $\varphi(r)=1$. 
Now we analyse two cases. The first case is when $\varphi(\infty)$ is equal to 0 , then returning to the original minimizer $u$, the monotonicity says that $\varphi(r)=0$ all the time, thus $\nabla u=0$ and $K$ locally minimizes the length $\mathcal{H}^{1}$ among topological competitors. This implies that $K$ must be one of the sets described in 1. 2. and 3. of the statement.

The most delicate case is when $\varphi(\infty)=1$. First notice that this value does not change by changing the origin (i.e. the point at which balls are centered in the computation of $\varphi$ ). Then, by a non trivial contradiction argument, it is possible to find in $K$ a point at witch $\varphi(0)=1$. Looking now at this point we obtain that $\varphi(r)$ is constant, equal to 1 , and the same argument as the one used just before says that $u$ is of the type described in 4 .

One way to prove the Mumford-Shah conjecture would be to improve the result of Theorem 3.3. Some work in this direction has been done in [DL02] (see Section 3.6 for more detail).

Open problem 3.4 (Implies the Mumford-Shah conjecture). Prove the same statement as Theorem 3.3 without assuming connectedness of $K$.

Elements of proof for Theorem 3.1. Let us describe the ingredients to deduce Theorem 3.1 from the classification of blow-up limits. One first thing is to prove the finite number of pieces. For this purpose Bonnet first proves that, even if the blowup limit at one point may not be unique in general, it is always of same type, which allows him to classify points with respect to the type of the blow-ups (regular point, cracktip, or triple point). Then he is able to prove that there is a finite number of triple points. Indeed, assuming that a sequence of triple points $T_{n}$ accumulates onto some point $P$, he gets a contradiction by considering, basically, the blow up limit in $B\left(T_{n}, 2\left|T_{n}-T_{n+1}\right|\right)$ which would converge to some fancy global minimizer with two triple points. Indeed, it is not difficult to see that a blow-up limit with "moving" center will also converge to one of the short list of global minimizer. Now if a sequence of triple points accumulates, one can construct a certain blow-up (with "moving center", centered at the sequence of triple points) whose density at the limit does not correspond to any of the list of Theorem 3.3, because it would have a density of two triple points (in reality the blow-up sequence $B\left(T_{n}, 2\left|T_{n}-T_{n+1}\right|\right.$ ) may not really work because one should also take into consideration the speed of convergence to the respective propeller at $T_{n}$ and $T_{n+1}$, but the idea is roughly the same). This implies the finite number of endpoints as well.

Then the $C^{1}$-regularity relies on the fact that, if $x_{0}$ is a point at which the blow-up limits are a lines, then there exits $r_{0}$ such that $K$ is almost flat in $B\left(x_{0}, r\right)$ for all $0<r<r_{0}$ and intersects $\partial B\left(x_{0}, r\right)$ on both sides for many $r>0$. In this situation the monotonicity Lemma can be straighten with a greater power, which implies that $r \mapsto \int_{B\left(x_{0}, r\right)}|\nabla u|^{2} d x$ behaves like $C r^{1+\alpha}$ (because the arc of circles are smaller, which lead to a better Poincaré-Wirtinger constant). This, morally says that $K$ is 
an almost-minimizing set for $\mathcal{H}^{1}$, with excess of minimality controlled by $C r^{1+\alpha}$. It is then classical to get $C^{1, \alpha / 2}$ estimates from this fact.

Remark 3.5. Bonnet also studies the triple points, at which he proves the existence of half-tangents for the three pieces of curves (thus uniqueness of blow-up at the triple point), whose tangents making angles of 120 degrees. A similar description is still an open problem for the cracktip.

Open problem 3.6 (Asked in page 571 of [Dav05b]). Prove (or disprove) the uniqueness of blow-up limit at an endpoint of $K$, with $C^{1}$ regularity up to the tip.

Up to our knowledge, the best regularity obtained at the tip of an endpoint for a Mumford-Shah minimizer is some logarithmic spiral estimates (see [Dav05b, G.69]).

\subsubsection{Three other $C^{1}$-Regularity results}

In what follows we try to present three other $C^{1}$ regularity results stated in a way that we can compare them.

We first introduce the normalized Hausdorff distance in $B(x, r)$ defined by

$$
\begin{aligned}
D_{x, r}(E, F) & :=\frac{1}{r}\left\{\max \left\{\sup _{y \in E \cap B(x, r)} d(y, F), \sup _{y \in F \cap B(x, r)} d(y, E)\right\}\right\} \\
& =\frac{1}{r} d_{H}(E \cap B(x, r), F \cap B(x, r)) .
\end{aligned}
$$

In [Dav96] (and re-written in the book [Dav05b] in a simplified way) the following $C^{1}$ result has been proved.

Theorem 3.7 ([Dav96]). Let $N=2$ and let $(u, K)$ be a reduced Mumford-Shah minimizer with gauge function $h(r)=C r^{\beta}$. Then there exists $\varepsilon, r_{0}>0$ and $c, \alpha \in$ $(0,1)$ such that whenever $x_{0} \in K$ and $0<r<r_{0}$ are such that $B\left(x_{0}, r\right) \subset \Omega$ and

$$
D_{x, r}(K, L) \leq \varepsilon
$$

for some line $L$, then $K \cap B(x, c r)$ is a $C^{1, \alpha}$ regular curve.

To be precise the statement of Theorem 3.7 is coming from [Dav05b, Corollary 17 ], except that in the latter statement a control on the energy of $u$ in $B(x, r)$ is also needed. But one can get rid of it by use of [Dav05b, Lemma 3 page 476] which says that, by a compactness argument, a small enough flatness implies a small energy as well, leading to Theorem 3.7.

Theorem 3.7 implies $C^{1}$ a.e. because the rectifiability of $K$ implies that (3.8) occurs at a.e. point $x$. Actually from the uniform rectifiability of $K$ we can even 
get a more accurate result, saying that in any ball $B(x, r)$ with $r \leq r_{0}$ there exists a ball $B(y, c r) \subset B(x, r)$ in which $K$ is $C^{1, \alpha}$. This also leads to the fact that $K$ is $C^{1, \alpha}$ outside a set of dimension $d<1$ (see [Dav96]).

Using the same technics, David also established a perturbation result around triple points. This was stated in [Dav96] with only a sketch of proof, and then written in [Dav05b] with slightly more detail. Let us recall that a propeller is the union of three half lines in the plane meeting at their tip by 120 degree angles.

Theorem 3.8 ([Dav96]). Let $N=2$ and let $(u, K)$ be a reduced Mumford-Shah minimizer with gauge function $h(r)=C r^{\beta}$. Then there exists $\varepsilon, r_{0}>0$ and $c, \alpha \in$ $(0,1)$ such that whenever $x_{0} \in K$ and $0<r<r_{0}$ are such that $B\left(x_{0}, r\right) \subset \Omega$ and

$$
D_{x, r}(K, P) \leq \varepsilon
$$

for some propeller $P$, then $K \cap B(x, c r)$ is a union of three $C^{1, \alpha}$ regular curves meeting at their tip by 120 degree angles.

The result of David only holds in dimension 2. For higher dimensions, there exists another famous result by Ambrosio, Fusco and Pallara [AP97, AFP97], re-written in the book [AFP00]. Here is a statement.

Theorem 3.9 ([AFP97]). Let $(u, K)$ be a reduced Mumford-Shah minimizer with gauge function $h(r)=C r^{\beta}$ (in any dimension). Then there exists $\varepsilon, r_{0}>0$ and $c, \alpha \in(0,1)$ such that whenever $x_{0} \in K$ and $0<r<r_{0}$ are such that $B\left(x_{0}, r\right) \subset \Omega$ and

$$
D_{x, r}(K, P) \leq \varepsilon
$$

for some hyperplane $P$, then $K \cap B(x, c r)$ is a $C^{1, \alpha}$ hypersurface.

Again, since we tried to make an analogy between different results, the statement of Theorem 3.9 is a bit different from its original version in [AFP97], but could be adapted. Originally, it was stated in an SBV fashion, the flatness is not exactly the same as our condition involving $D_{x, r}$ but is comparable (actually the one that appears later in (3.20)), and a condition on the normalized energy of $u$ is also needed in [AFP97], which can be removed via a compactness argument similar to the one of [Dav05b, Lemma 3 page 476] (see also later the discussion in the paragraph just after the statement of Theorem 3.22).

The proof of Theorem 3.9 relies on a key variational estimate, the so called "tilt estimate", which measures the oscillation of approximative tangent planes with respect to a fixed plane in a ball. This estimate is strongly well-adapted for a flat situation only, and the same proof could not be used for obtaining other perturbation results like the 2D one of David near propeller (Theorem 3.8), or its possible generalization in dimension 3. 
This was done later in [Lem08], using a different approach. We recall that a minimal cone in $\mathbb{R}^{3}$ is a set belonging to the following list (see also Section 3.4 below).

- A plane (cone of type $\mathbb{P})$.

- A union of three half-planes meeting along their edges by 120 degree angles

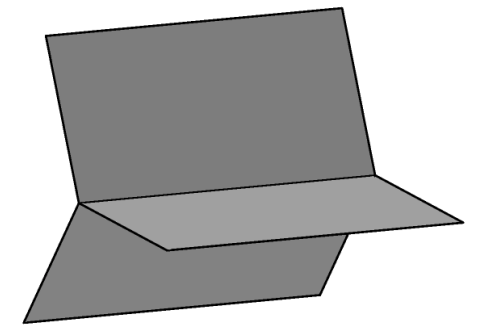

(cone of type $\mathbb{Y}$ ).

- A cone over the union of the edges of a regular tetrahedron (cone of type $\mathbb{T}$ ).

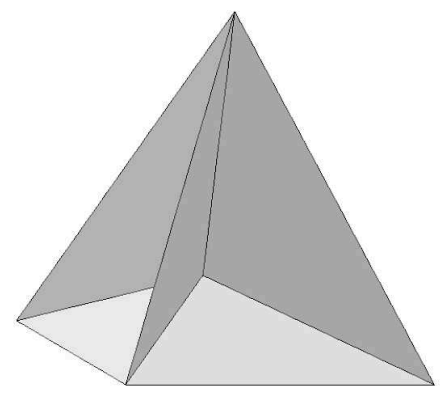

Theorem 3.10 ([Lem11] or [Lem08]). Let $N=3$ and let $(u, K)$ be a reduced Mumford-Shah minimizer with gauge function $h(r)=C r^{\beta}$. Then there exists $\varepsilon, r_{0}>$ 
0 and $c, \alpha \in(0,1)$ such that whenever $x_{0} \in K$ and $0<r<r_{0}$ are such that $B\left(x_{0}, r\right) \subset \Omega$ and

$$
D_{x_{0}, r}(K, Z) \leq \varepsilon
$$

for some minimal cone $Z$ of type $\mathbb{P}, \mathbb{Y}$ or $\mathbb{T}$ centered at point $x_{0}$, then there exists a diffeomorphism $\phi$ of class $C^{1, \alpha}$ from $B\left(x_{0}, c r\right)$ to its image, verifying $\phi(0)=x_{0}$, $\left|\phi(y)-\left(y+x_{0}\right)\right| \leq 10^{-3}$ cr, and such that

$$
\phi(K) \cap B\left(x_{0}, c r\right)=Z \cap B\left(x_{0}, c r\right) .
$$

The proof of Theorem 3.10 will be explained in Section 3.5.

Another version of this theorem obtained from the preceding one and using blowup limits, is the following.

Theorem 3.11 ([Lem11], Theorem 9). Let $N=3$ and let $(u, K)$ be a reduced Mumford-Shah minimizer with gauge function $h(r)=C r^{\beta}$. Then there exists $\varepsilon, r_{0}>$ 0 and $c, \alpha \in(0,1)$ such that the following holds. Assume that $x_{0} \in K$ and $0<r<r_{0}$ are such that $B\left(x_{0}, r\right) \subset \Omega$ and

$$
\frac{1}{r^{2}} \int_{B\left(x_{0}, r\right) \backslash K}|\nabla u|^{2} d x \leq \varepsilon .
$$

Then there is a diffeomorphism $\phi$ of class $C^{1, \alpha}$ from $B(0, c r)$ to its image, verifying $\phi(0)=x_{0},\left|\phi(y)-\left(y+x_{0}\right)\right| \leq 10^{-3} c r$, and there is a minimal cone $Z$ such that $K \cap B\left(x_{0}, c r\right)=\phi(Z) \cap B\left(x_{0}, c r\right)$.

\subsection{Almost minimal sets in $\mathbb{R}^{3}$ and Jean Taylor's Theorem}

The proof of Theorem 3.10 uses, as a very first step, the local description of almost minimal sets in $\mathbb{R}^{3}$ studied by Taylor [Tay76], which says that they are locally $C^{1}$ equivalent to a minimal cone. Those results were originally stated in the language of currents for a notion of minimizers introduced by Almgren. More recently, David established the same type of results with a longer but elementary proof in [Dav09, Dav10], extending some of the statements in higher dimensions, and working in particular with another class of minimizers that he called MS-minimal sets. The main point about MS-minimal sets is that they are very well adapted to MumfordShah minimizers. In particular, if $(u, K)$ is a Mumford-Shah minimizer with $\nabla u=0$ then $K$ is an almost MS-minimal set in the sense of [Dav09]. Actually, it can even be an equivalent Definition, and we find it convenient here to proceed this way.

Definition 3.12. A set $K$ is a MS-almost minimal set with gauge function $h(r)$ if $(K, 0)$ is an almost Mumford-Shah minimizer with gauge function $h(r)$ in the sense of Definition 2.4. 
The main difference with the definition of Almgren, is essentially the class of competitors that are any closed set satisfying the topological condition for MSminimal sets (i.e. preserving the separation of pair of points outside the competing ball), instead of being images of a given set by a family of continuous mappings in Almgren's definition (one implies the other actually). One of the main result in [Dav10] is the following.

Theorem 3.13 ([Dav10]). Let $N=3$ and let $K$ be a reduced almost MS-minimal set with gauge function $h(r)=C r^{\beta}$. Then there exists $\varepsilon, r_{0}>0$ and $c, \alpha \in(0,1)$ such that whenever $x_{0} \in K$ and $0<r<r_{0}$ are such that $B\left(x_{0}, r\right) \subset \Omega$ and

$$
D_{x_{0}, r}(K, Z) \leq \varepsilon
$$

for some minimal cone $Z$ of type $\mathbb{P}, \mathbb{Y}$ or $\mathbb{T}$ centered at point $x_{0}$, then there exists a diffeomorphism $\phi$ of class $C^{1, \alpha}$ from $B\left(x_{0}, c r\right)$ to its image, verifying $\phi(0)=x_{0}$, $\left|\phi(y)-\left(y+x_{0}\right)\right| \leq 10^{-3} \mathrm{cr}$, and such that

$$
\phi(K) \cap B\left(x_{0}, c r\right)=Z \cap B\left(x_{0}, c r\right) .
$$

As we can notice, the difference with Theorem 3.10 is passing from MS-almost minimal sets to Mumford-Shah minimizers, and this will be explained in the next section.

Another interesting result is the following one, regarding to global MS-minimal sets in $\mathbb{R}^{3}$.

Theorem 3.14 ([Dav09] Theorem 1.9). Let $K \subset \mathbb{R}^{3}$ be a MS-almost minimal set in $\mathbb{R}^{3}$ with gauge function $h=0$. Then $K$ is a minimal cone of type $\mathbb{P}, \mathbb{Y}$, or $\mathbb{T}$.

The little surprise concerning Theorem 3.14 is that no analogous result is known for minimal sets in the sense of Almgren. Curiously enough, the topological condition used in the definition of competitors for MS-minimal sets and coming from the theory of Mumford-Shah minimizers, is crucial to get the result.

Indeed, the proof of Theorem 3.14 relies on the monotonicity behavior of the density $r \mapsto \mathcal{H}^{2}(K \cap B(x, r)) / r^{2}$, for any $x \in K$, and the fact that constant density implies that $K$ is a cone. Therefore, the game is to find a point $x \in K$ which has the same density when $r \rightarrow 0$ as the one when $r \rightarrow+\infty$ in order to obtain a constant density (by monotonicity) and conclude that $K$ is a cone. Then we would know that it is a $\mathbb{P}, \mathbb{Y}$ or $\mathbb{T}$ thanks to Jean Taylor [Tay76]. If the density at $r \rightarrow+\infty$ is the same as a cone $\mathbb{P}$, then it is very easy to find a "regular point" (thanks to the regularity theory for minimal sets) $x \in K$ with the same density as $r \rightarrow 0$ and thus concluding that $K$ is a cone of type $\mathbb{P}$. If the density at $r \rightarrow+\infty$ is the one of a $\mathbb{Y}$, then it is a little more delicate but doable to find a point $x \in K$ with the same density and conclude that $K$ is of type $\mathbb{Y}$. Finally, if the density at $r \rightarrow+\infty$ is 
the one of a cone of type $\mathbb{T}$, then the situation becomes technically more involved. David is indeed able to find a point $x \in K$ of type $\mathbb{T}$ but the proof really needs some non trivial topological arguments, in particular uses $K$ to be a MS-minimal set (i.e. with topological condition for the class of competitors).

\subsection{Sketch of proof for the $C^{1}$ regularity in $\mathbb{R}^{3}$}

In this section we would like to present the main ideas and steps of the proof of Theorem 3.10. The presentation here follows closely the one written in french in [Lem12].

Thus, let $(u, K)$ be a minimizer that we suppose very close to a minimal cone in $B\left(x_{0}, r_{0}\right)$. In other words,

$$
D_{x_{0}, r_{0}}(K, Z) \leq \varepsilon
$$

for a certain minimal cone $Z$ centered at $x_{0}$ of type $\mathbb{P}, \mathbb{Y}$ or $\mathbb{T}$, and for some $\varepsilon>0$ which during all the proof could be taken as small as we want.

One of the essential ingredient which will be omitted here is a quantity called the normalized jump of $u$, which controls the size of "holes" in the set $K$. To simplify, we will assume that $K$ is separating $B\left(x_{0}, r_{0}\right)$ into at least as many big connected components as does the minimal cone $Z$. Using a similar argument already used in [Dav05b], we can reduce to this case by adding to $K$ some pieces of level sets of the function $u$, and control the measure of what we added by the coarea formula. We refer to [Lem11] and do not wish to enter into more detail here.

To simplify a little more we will also assume that $h=0$, the general case follows by the same way except that we add everywhere a pertubating term of type $r^{2} h(r)$ in all the estimates.

The main strategy is to control the normalized energy of $u$, prove that it decays like a power of the radius in order to obtain that $K$ is actually an almost minimal set and apply David's version of Taylor's theorem to it (see Section 3.4). While some compactness argument will be used to control the energy of $u$, another technic perhaps more original in the context of Mumford-Shah minimizers will appear, which uses a geometrical stopping time on the flatness (or more generally "closeness to a cone") to construct new competitors and produce interesting estimates.

To understand the behavior of $u$ we first consider the case of a harmonic function.

\subsubsection{Decay of energy for harmonic functions}

The fundamental general question to solve is the following : let $K \subset B(0,1)$ be a closed set and $u \in W^{1,2}(B(0,1) \backslash K)$ a harmonic function with Neumann boundary condition on $K$ (in the weak sense, i.e. $u$ minimizes locally the Dirichlet energy 
$\left.\int_{B(0,1) \backslash K}|\nabla u|^{2} d x\right)$. Is it true that

$$
\int_{B\left(0, \frac{1}{2}\right) \backslash K}|\nabla u|^{2} d x \leq\left(\frac{1}{2}\right)^{N-1+\eta} \int_{B(0,1) \backslash K}|\nabla u|^{2} d x,
$$

for some $\eta>0$ ?

It is easy to construct examples of sets $K$ for which (3.14) is not verified, including examples of smooth and very flat $K$, for instance when $K$ is a little tube: the union of two lines very close to each other in $\mathbb{R}^{2}$. Let us see this more precisely. To simplify, let us assume that $B(0,1)$ is a square: it does not change much for the purpose of what we want to explain, and the computations will be very easy. Let's consider $K=\mathbb{R} \times\{0\} \cup(\mathbb{R}+\varepsilon) \times\{0\} \subset \mathbb{R}^{2}$, the union of two horizontal lines, $\varepsilon$-close to each other. Then $\partial B(0,1) \backslash K$ is divided into four regions, two big "arc of square" in the north and south direction (that we call $N$ and $S$ ), and two very small segments on est and west direction (called $E$ and $W$ respectively, the sides of the "tube"). Let us define a function $f$ on $\partial B(0,1) \backslash K$ which is locally constant, equal to zero everywhere, except on the est-side $E$, where it is equal to 2. It is most obvious to check that the Dirichlet minimizer $u$ in $B(0,1) \backslash K$ with that boundary data on $\partial B(0,1) \backslash K$ is an affine function going from 2 to 0 linearly in the horizontal direction in the small tube and vertically constant (because it is the unique harmonic function satisfying the Neumann boundary condition on $K \cap B(0,1)$ and matching the Dirichlet data on $\partial B(0,1) \backslash K)$. This function then satisfies $|\nabla u|=1$. Consequently, for all $r>\varepsilon$, we have

$$
\int_{B(0, r) \backslash K}|\nabla u|^{2} d x=4 r \varepsilon
$$

In other words the energy $\int_{B(0, r) \backslash K}|\nabla u|^{2} d x$ behaves linearly in $r$, and there is no hope of getting a improved decaying of energy in small balls like $r^{1+\eta}$, as desired for (3.14).

Conversely, here are some examples where it holds true.

1. If $K=\emptyset$, then (3.14) is true with $\eta=1$ : is it just the mean value inequality applied to the super-harmonic function $|\nabla u|^{2}$.

2. If $K$ is a hyperplane, then (3.14) is true : it suffice to use a reflection and apply the preceding case.

3. If $K$ is a minimal cone in $\mathbb{R}^{3}$, of type $\mathbb{Y}$ or $\mathbb{T}$, then (3.14) is still true. This follows from an estimate on the first eigenvalue of the Neumann-Laplace-Beltrami operator on $\partial B(0,1) \backslash Z$, where $Z$ is a minimal cone (see [Lem10]). Notice that a tentative proof with a reflection technic fails. 
4. If $K$ is $\varepsilon$-close to a minimal cone for the Hausdorff distance, and is an $\varepsilon_{0^{-}}$ minimal set (see the definition just below), then (3.14) is true.

Definition 3.15. We say that $K$ is $\varepsilon_{0}$-minimal if for all $x \in K$ and $r<r_{0}$ there exists a minimal cone $Z(x, r)$ of type $\mathbb{P}, \mathbb{Y}$ or $\mathbb{T}$ such that

$$
D_{x, r}(K, Z(x, r)) \leq \varepsilon_{0} .
$$

The $\varepsilon_{0}$-minimal sets introduced in [DDPT08] are built upon the same idea as the so-called Reifenberg-flat sets introduced by Reifenberg in the sixties. In particular, one proves that they are locally the bi-Hölder image of a minimal cone around each of their point. We will see later the link with a Mumford-Shah minimizer.

Point 4. above is the purpose of the paper [Lem10]. The proof works by "compactness" : we know that (3.14) is true when $K$ is a minimal cone, then it should still be true for any set "close" to a minimal cone for the Hausdorff distance otherwise we would get a contradiction by passing to the limit. The assumption of being an $\varepsilon_{0}$-minimal set is useful to pass to the limit. It guarantees the continuity of the Neumann problem, namely a sequence of functions $u_{n}$ such that $u_{n}$ is a Dirichlet minimizer in $B(0,1) \backslash K_{n}$ converges to a Dirichlet minimizer in $B(0,1) \backslash K$ when $K_{n}$ converges to $K$ for the Hausdorff distance, and provided that the $K_{n}$ are all $\varepsilon_{0}$-minimal (with the same $\varepsilon_{0}$ ).

Now to be totally honest, in reality we need a result more elaborate than the one we just said. Indeed, we will actually need the following slightly modified version of Point 4.

5. If $K$ is $\varepsilon$-close to a minimal cone in $B(0,1)$ and simultaneously $\varepsilon_{0}$-minimal except for a possible family of bad balls $\left\{B_{i}\right\}_{i \in I}$ centered on $K$, then (3.14) stays true if we replace $K$ by the new set $K \cup \bigcup_{i \in I^{*}} \partial B_{i}$ where

$$
I^{*}:=\left\{i \in I ; \partial B_{i} \cap \partial B(0,1 / 2) \neq \emptyset\right\} .
$$

The proof is similar to Point 4. with further technical complications. Morally, one can think that adding the set $\bigcup_{i \in I^{*}} \partial B_{i}$ is a good way to "cut the tubes" and prevent the counter example described above to occur. Now for what the proof is concerned, the main tool is a sort of Whitney extension argument. Namely, the argument is by compactness thus one needs to prove the convergence of Dirichlet minimizers for a sequence $K_{n}$ of $\varepsilon_{0}$-minimal sets that converge for the Hausdorff distance. In that procedure, one wants to compare the energy of two different Dirichlet minimizers $u_{n_{1}}$ and $u_{n_{2}}$ associated with two different sets $K_{n_{1}}$ and $K_{n_{2}}$. To do so, we construct a competitor $\tilde{u}_{n_{2}}$ for $u_{n_{1}}$ from $u_{n_{2}}$ by extending it near $K_{n_{1}}$ where it is possibly not well defined. This is done via a covering of $K_{n_{1}}$ by balls of size comparable 
to $\delta=\operatorname{dist}\left(K_{n_{1}}, K_{n_{2}}\right)$ and by extending $u_{n_{2}}$ by its average locally on each balls. The argument works thanks to the control on the geometry of $K_{n_{1}}$ in each ball which allows us to produce estimates: it is indeed flat in each ball thanks to the $\varepsilon_{0}$-minimality of $K_{n_{1}}$, provided that $\delta \leq \varepsilon_{0}$. There is only one difference concerning the bad balls $B_{i}$ for $i \in I^{*}$ : their radii cannot be $\delta$, as small as we want, but stay "large". But we don't care since the new function on which we get a result, i.e. the minimizer associated with $K \cup \bigcup_{i \in I^{*}} \partial B_{i}$ instead of just $K$, has zero energy is all the bad balls $B_{i}$ with $i \in I^{*}$. Of course later we will need to compare the energy of this new function with the original one, that minimizes in $B \backslash K$, and this will be done in terms of energy in the $B_{i}$, which can be small enough provided to take first a good radius that meet the least bad balls as much as possible in the definition of $I^{*}$.

\subsubsection{Geometric stopping time}

In Section 3.5.1 we have seen how to control the energy of a harmonic function when $K$ is $\varepsilon_{0}$-minimal. How to reduce to that in general? Answer : by a stopping time argument. Indeed, let $\varepsilon<\varepsilon_{0}$ be given and $(u, K)$ our Mumford-Shah minimizer which is supposed to be $\varepsilon$-close to a minimal cone in $B\left(x_{0}, 2 r_{0}\right)$. We define

$$
\beta(x, r):=\inf _{Z \text { minimal cone }} D_{x, r}(K, Z),
$$

and for all $x \in K \cap B\left(x_{0}, r_{0}\right)$

$$
d(x):=\inf \left\{s>0 ; \beta(x, t) \leq \varepsilon_{0} \forall t \in\left(s, r_{0}\right)\right\} .
$$

Since $\beta\left(x_{0}, 2 r_{0}\right) \leq \varepsilon$, and since we always have $\beta(x, \lambda s) \leq \frac{1}{\lambda} \beta(x, s)$, we know that $d(x) \leq \frac{\varepsilon}{\varepsilon_{0}}$ for all $x \in K \cap B\left(x_{0}, r_{0}\right)$. Finally, we define the "bass mass" as being

$$
m(x, r):=\frac{1}{r^{2}} \mathcal{H}^{2}\left(\bigcup_{y \in K \cap B(x, r)} K \cap B(y, d(y))\right) .
$$

If $\left\{B_{i}\right\}$ is a Vitali covering of $\{B(x, d(x))\}$, then $K \cap B\left(x_{0}, r_{0}\right)$ is an $\varepsilon_{0}$-minimal set in $K \backslash \bigcup_{i} 2 B_{i}$. The important fact is that, since $K \cap B\left(x_{0}, r_{0}\right)$ is an $\varepsilon_{0}$-minimal set in $K \backslash \bigcup_{i} 2 B_{i}$, we could apply Point 5 above to estimate the energy of $u$. But before that, let us see how to control the quantity $m(x, r)$.

\subsubsection{Estimate on the bad balls}

The control of the quantity $m(x, r)$ is achieved via a compactness argument, and the well-known fact [Dav10, Corollary 12.25] that for an almost minimal set, the quantity $\beta(x, r)$ defined in (3.15) behaves like $C r^{\alpha}$ due to the regularity theory of almost minimal sets. 
If $r:=d(x)>0$ for some $x \in K$, then by definition $\beta(x, 2 r) \leq \varepsilon_{0}$ and $\beta(x, r)>\varepsilon_{0}$. Thus $K \cap B(x, 2 r)$ cannot be a minimal set (because in this case $\beta(x, r)$ would decay like $\left.C r^{\alpha}\right)$. We deduce that there exists some competitor $L$ such that $\mathcal{H}^{2}(L \cap$ $B(x, 2 r))<\mathcal{H}^{2}(K \cap B(x, 2 r))$. We can even make this estimate quantitative, namely $\mathcal{H}^{2}(L \cap B(x, 2 r)) \leq \mathcal{H}^{2}(K \cap B(x, 2 r))-\delta r^{2}$ with some $\delta>0$ which depends on $\varepsilon_{0}$.

Next by modifying each "bad ball" $B_{i}$ with the competitor $L$ found just above, and assuming that one can define a suitable extension of $u$ in each of those balls $B_{i}$ modified by this way (which will be explained just after), we obtain that the total mass of the "bad balls" is controlled by the minimality defect of $K$, which itself is controlled by the energy of $u$. In other words we have obtained an estimate of type

$$
m\left(x_{0}, r_{0}\right) \leq \frac{C}{\delta}\left(\frac{1}{r_{0}^{2}} \int_{B\left(x, r_{0}\right)}|\nabla u|^{2} d x\right)
$$

which is one of the key estimates.

Remark 3.16. In fact the estimate (3.16) is not totally true as it is written, because of "boundary effects". Indeed, we cannot replace $K$ by $L$ in the balls $B_{i}$ that touch $\partial B\left(x_{0}, r_{0}\right)$. Consequently we do not control really $m\left(x_{0}, r_{0}\right)$ but $m\left(x_{0}, r_{0}\right)$ minus the total mass of balls $B_{i}$ that touch $\partial B\left(x_{0}, r_{0}\right)$. This brings some more technical complication but can be solved by chosing a "good sphere" that touches the less balls $B_{i}$ as possible.

\subsubsection{Extension of Whitney type}

We saw in the preceding section that we may need to define a competitor for the function $u$ in the union of all bad balls $B_{i}$, where $K$ has been modified. To do so, we use a "Whitney type" extension, which allows to construct a new function well defined outside our new set $K^{\prime}$, coinciding with $u$ outside all the $2 B_{i}$ and without loosing too much energy. The key point is that we always consider balls $B(x, r)$ with $r \geq d(x)$. By this way, the geometry of the set is always under control: there $K$ is $\varepsilon_{0}$-close to a minimal cone. Therefore we can use a partition of unity and define our new function in each ball $B(x, r)$ using some averages of $u$ in balls of radius equivalent to $r$ in big connected components of $B(x, r) \backslash K$ (see [Lem10, Lemma 17]).

The above procedure is used several times to find a suitably well defined function $\tilde{u}$ in the complement of a set $\tilde{K}$, which is a competitor for $K$ obtained using the stooping balls $B_{i}$. The crucial point is that the extended function $\tilde{u}$ verifies

$$
\int_{B\left(x_{0}, r_{0}\right) \backslash \tilde{K}}|\nabla \tilde{u}|^{2} \leq \int_{B\left(x_{0}, r_{0}\right) \backslash K}|\nabla u|^{2}+C \int_{R}|\nabla u|^{2},
$$

where $R$ is the region where $\tilde{u}$ and $u$ differ. 


\subsubsection{Control of normalized energy for a Mumford-Shah minimizer}

We now arrive to the second fundamental estimate, the one about the normalized energy when the singular set is close to a minimal cone.

Let $r<r_{0}$ and let $I^{*} \subset I$ be the indices of all the bad balls $B_{i}$ that meet $\partial B\left(x_{0}, r\right)$. Let $K^{\prime}=K \cup \bigcup_{i \in I^{*}} \partial B_{i}$ and let $v$ be the harmonic function in $B\left(x_{0}, r_{0}\right) \backslash K^{\prime}$ that is equal to $u$ on $\partial B\left(x_{0}, r_{0}\right)$ with Neumann conditions on $K^{\prime}$. Then from Section 3.5.1 we know how to control the energy of $v$. Thus is suffice to compare $\left(v, K^{\prime}\right)$ and $(u, K)$ in $B\left(x_{0}, r_{0}\right)$. Since $(u, K)$ is a Mumford-Shah minimizer,

$$
\begin{aligned}
\int_{B\left(x_{0}, r_{0}\right) \backslash K}|\nabla u|^{2} d x & \leq \int_{B\left(x_{0}, r_{0}\right) \backslash K^{\prime}}|\nabla v|^{2} d x+\mathcal{H}^{2}\left(K^{\prime}\right)-\mathcal{H}^{2}(K) \\
& \leq \int_{B\left(x_{0}, r_{0}\right) \backslash K^{\prime}}|\nabla v|^{2} d x+r^{2} m^{\prime}\left(x_{0}, r\right)
\end{aligned}
$$

where $m^{\prime}\left(x_{0}, r\right)=\frac{1}{r^{2}} \mathcal{H}^{2}\left(\bigcup_{i \in I^{*}} \partial B_{i}\right)$.

On the other hand $u$ is a competitor for $v$ as an energy minimizer, thus $\nabla v$ and $\nabla(u-v)$ are orthogonal in $L^{2}$, and by Pythagoras we have

$$
\int_{B\left(x_{0}, r_{0}\right) \backslash\left(K \cup K^{\prime}\right)}|\nabla v-\nabla u|^{2}=\int_{B\left(x_{0}, r_{0}\right) \backslash K}|\nabla u|^{2}-\int_{B\left(x_{0}, r_{0}\right) \backslash K^{\prime}}|\nabla v|^{2} \leq r^{2} m^{\prime}\left(x_{0}, r\right) .
$$

Which yields,

$$
\begin{aligned}
\int_{B\left(x_{0}, r\right) \backslash K}|\nabla u|^{2} & \leq 2 \int_{B\left(x_{0}, r\right) \backslash K^{\prime}}|\nabla v|^{2}+2 \int_{B\left(x_{0}, r\right) \backslash\left(K \cup K^{\prime}\right)}|\nabla v-\nabla u|^{2} \\
& \leq 2\left(\frac{r}{r_{0}}\right)^{2+\eta} \int_{B\left(x_{0}, r_{0}\right) \backslash K^{\prime}}|\nabla v|^{2}+2 \int_{B\left(x_{0}, r_{0}\right) \backslash\left(K \cup K^{\prime}\right)}|\nabla v-\nabla u|^{2} \\
& \leq 2\left(\frac{r}{r_{0}}\right)^{2+\eta}\left(\int_{B\left(x_{0}, r_{0}\right) \backslash K}|\nabla u|^{2}\right)+2 r^{2} m^{\prime}(x, r)
\end{aligned}
$$

which is our fundamental estimate about normalized energy.

\subsubsection{Conclusion}

By choosing a suitable radius $r$ in Section 3.5.5 that meets as little bad balls as possible, we can show that (3.17) implies

$$
\int_{B\left(x_{0}, r\right) \backslash K}|\nabla u|^{2} \leq 2\left(\frac{r}{r_{0}}\right)^{2+\eta}\left(\int_{B\left(x_{0}, r_{0}\right) \backslash K}|\nabla u|^{2}\right)+2 \varepsilon r^{2} m(x, r) .
$$

Iterating this last estimate together with (3.16) yields $\int_{B\left(x_{0}, r\right) \backslash K}|\nabla u|^{2} \leq C r^{2+\eta}$. The same estimate holds true for all the $x$ in $K \cap B\left(x_{0}, r_{0}\right)$ which is enough to conclude that $K$ is an almost minimal set in $B\left(x_{0}, c r_{0}\right)$ where $c$ is a universal constant, which lead to the conclusion of the Theorem. 


\subsection{The David-Léger paper: a little closer to the conjecture}

As we said in Open Problem 3.4, a way to prove the Mumford-Shah conjecture would be to classify the possible blow-up limits (i.e. Theorem 3.3) without assuming that $K$ is connected. In [DL02], some investigation in that direction was made which led to the following result.

Theorem 3.17. [DL02] If $(u, K)$ is a reduced global minimizer in $\mathbb{R}^{2}$ and if $K$ is neither a line, nor a propeller, then $\mathbb{R}^{2} \backslash K$ is connected.

This means that to find a possible extra global minimizer, one may assume that $K$ is not connected and $\mathbb{R}^{2} \backslash K$ is connected.

But a nice corollary of Theorem 3.17 is also that it links the Mumford-Shah conjecture with another famous conjecture by De Giorgi.

Open problem 3.18 (De Giorgi, 1989). Prove that the cracktip function

$$
\left.u(r, \theta)=\sqrt{\frac{2}{\pi}} r \sin (\theta / 2), r>0, \theta \in\right]-\pi, \pi[,
$$

is the only non constant function verifying

$$
\int_{B}|\nabla u|^{2} d x+\mathcal{H}^{1}\left(S_{u} \cap B\right) \leq \int_{B}|\nabla v|^{2} d x+\mathcal{H}^{1}\left(S_{v} \cap B\right)
$$

for any $B \subset \mathbb{R}^{2}$ and any $v \in S B V(B)$ satisfying $v=u$ in $\mathbb{R}^{2} \backslash B$, up to additional constant, translation or rotation.

Observe that no topological condition on competitors is assumed in the above minimizing problem. But when $\mathbb{R}^{2} \backslash K$ is connected then the topological constraint on competitor is automatically satisfied. Therefore, as a consequence of Theorem 3.17 (and together with Bonnet's Theorem) the Mumford-Shah conjecture reduces to the question of De Giorgi.

\subsection{The nice formula of Léger and consequences}

About global minimizers in the plane, there is a very nice formula from [Leg99] which have, curiously enough, very few application (up to now). If $(u, K)$ is a global minimizer in $\mathbb{R}^{2}$ then

$$
\left(\frac{\partial u}{\partial z}\right)^{2}=-\frac{1}{8 \pi} \int_{K} \frac{1}{(z-w)^{2}} d \mathcal{H}^{1}(w),
$$

where

$$
\frac{\partial u}{\partial z}=\frac{1}{2}\left(\frac{\partial u}{\partial x}-i \frac{\partial u}{\partial y}\right)
$$


The formula comes from the Euler-Lagrange equation of first order, with a careful interpretation in terms of complex analysis. Among interesting consequences of this formula, one can recover the necessary constant $\sqrt{2 / \pi}$ in front of the cracktip function. Notice also that it implies the fact that $u$ is uniquely determined by the set $K$ (up to multiplicative sign and additional constants). This is still an open question in higher dimensions. Another interesting result is the following one.

Proposition 3.19. [Leg99, Proposition 25] If $(u, K)$ is a reduced global minimizer, and $K$ is contained in a line, then $K$ is either a line, a half-line, or the empty-set.

What is remarkable in the above statement is that it has no connectedness assumption on $K$ a priori. In particular the monotonicity of Bonnet does not apply in this situation. It is the only statement of that kind that I know for which a classification of global minimizers is concluded without assuming any topological constraint on $K$ (however, being contained in a line looks a bit restrictive). A similar statement in higher dimension is still an open question, for which a partial result is given in Section 4.3.

Open problem 3.20 (Asked on page 442 and again on page 571 in [Dav05b]). Prove that, if $(u, K)$ is a reduced global minimizer in $\mathbb{R}^{3}$ and $K$ is contained in a plane, then $K$ is either a plane, a half-plane or the empty-set.

\subsection{Dimension of the Singular set and higher integrability of the gradient}

After the local regularity results mentioned earlier, a natural question is wether one can say something on the size of the singular set. More precisely, for a minimizer $(u, K)$ one can define the (closed) set

$$
\Sigma:=\left\{x \in K ; K \cap B(x, r) \text { is never a } C^{1, \alpha} \text { hypersurface, for any } r\right\} .
$$

What can be conjectured is the following.

Open problem 3.21. Prove that $\mathcal{H}-\operatorname{dim}(\Sigma) \leq N-2$.

Up to know this question is still open. The best partial result toward the conjecture is the following.

Theorem 3.22 ([Dav96, Rig00, MS01, DLF13, DPF14]). If $(u, K)$ is a minimizer then $\mathcal{H}-\operatorname{dim}(\Sigma)<N-1$.

Let us say a few words about the different contributions to this theorem. It was first obtained by David [Dav96] in dimension 2. The idea is to use a quantitative 
version of the $\varepsilon$-regularity result. Indeed, recall that the regularity results (see Section 3.3.2) are usually of the following type: if some quantities are small enough in some ball, then $K$ is $C^{1, \alpha}$ in the half ball. Then different statements can be derived depending on which quantity you prefer to be small. It can be the (bilateral)- $\beta_{\infty}$ number alone, as we have chosen in the statements of Section 3.3.2),

$$
\inf _{P \text { hyperplane }} \frac{1}{r}\left(\sup _{x \in K \cap B(x, r)} \operatorname{dist}(x, P)+\sup _{x \in P \cap B(x, r)} \operatorname{dist}(x, K)\right) \leq \varepsilon_{1},
$$

or it can be the "unilateral" $\beta_{\infty}$ plus normalized energy,

$$
\left(\inf _{P \text { hyperplane }} \frac{1}{r} \sup _{x \in K \cap B(x, r)} \operatorname{dist}(x, P)\right)+\frac{1}{r^{N-1}} \int_{B_{r}}|\nabla u|^{2} d x \leq \varepsilon_{2},
$$

or it can also be its $L^{2}$ version "unilateral" $\beta_{2}$ plus normalized energy like in the original statement of [AFP97]

$$
\inf _{P \text { hyperplane }}\left(\frac{1}{r^{N-1}} \int_{K \cap B(x, r)}\left(\frac{1}{r} \operatorname{dist}(y, P)\right)^{2} d y\right)^{\frac{1}{2}}+\frac{1}{r^{N-1}} \int_{B_{r}}|\nabla u|^{2} d x \leq \varepsilon_{3} .
$$

Any of the three conditions (3.18), (3.19), or (3.20) would imply that $K \cap B(x, r / 2)$ is $C^{1, \alpha}$ smooth provided that the $\varepsilon_{i}$ are small enough ${ }^{4}$. Actually, it can be shown that each condition implies the others, up to fix the $\varepsilon_{i}$ accordingly and possibly changing the radius $r$ by $r / 2$ (the implication $(3.19) \Rightarrow(3.20)$ being indeed obvious, the others need some work).

Subsequently, from the rectifiability of $K$ it is not difficult to prove that

$$
\mathcal{H}^{N-1}(\Sigma)=0
$$

because $K$ admits an approximative tangent plane $\mathcal{H}^{N-1}$-a.e., which turns out to be a true tangent plane (because it is Ahlfors-regular) so that (3.18) occurs $\mathcal{H}^{N-1}$-a.e.

Now the main idea behind the approach in [Dav96] for a proof of Theorem 3.22 is to strenghten this argument using the uniform rectifiability of $K$ instead of just rectifiability. It is then possible to show, via a Carlson measure estimate, that for instance (3.19) occurs in many balls, in a quantified way. More precisely, for any ball $B(y, t) \subset \Omega$ with $y \in K$ and $t \leq r_{0}$, there exists some $B(x, r) \subset B(y, t)$ with $r \geq C t$ for which (3.19) holds true (this is also related to the notion of "porosity" that was used later in [DPF14], see next section). Using that fact, David was able to prove $\mathcal{H}-\operatorname{dim}(\Sigma)<N-1$ by a covering argument and a decomposition into dyadic cubes.

\footnotetext{
${ }^{4}$ for the record, (3.20) would be denoted by $\beta_{K, 2}(x, r)+\omega_{2}(x, r) \leq \varepsilon_{3}$ according to the notation of [Dav05b] and by $\mathcal{A}(x, r)+\mathcal{D}(x, r) \leq \varepsilon_{3}$ according to the notation of [AFP00].
} 
It was then extended in higher dimensions by Rigot [Rig00] using the same approach and the regularity result of [AFP97] as a replacement for the 2 dimensional one of [Dav96]. At the same time, and independently from [Rig00], Maddalena and Solimini [MS01] obtained the same result with an approach which was not so far from Rigot but where some technics introduced by the same authors in previous works were more exploited. Many years later, an alternative proof by De Philippis and Figalli appeared as a by product of [DPF14] and [AFH03], based again, in some sense, on the uniform rectifiability of $K$ (that the authors rephrased through the notion of "porosity").

Let us give some details about this last contribution. Yet, another interesting and related question is the higher integrability of the gradient of $u$. Indeed, a priori we only know that $\nabla u \in L^{2}(\Omega)$. On the other hand, a simple computation on the cracktip function $\sqrt{\frac{2 r}{\pi}} \sin (\theta / 2)$ shows that it belongs to $L^{p}(B(0,1))$ for any $p<4$. Therefore, if we believe to the Mumford-Shah conjecture, then the worst possible singularity for a minimizer $u$ would be around crack-tips, and thus $\nabla u$ would, locally in $\Omega$, belong to all the $L^{p}$ for $p<4$.

Open problem 3.23 (Implied by the Mumford-Shah conjecture). If ( $u, K)$ is a minimizer in $\Omega \subset \mathbb{R}^{2}$ then $\nabla u \in L_{l o c}^{p}(\Omega)$ for all $p<4$.

Very recently, some gain of interest concerning this question has been brought back in [DLF13, DPF14], where the authors got some partial advance, by-passing the Mumford-Shah conjecture. Taking advantage of the known $\varepsilon$-regularity results, they were able to prove the following.

Theorem 3.24 ([DLF13] for $N=2$, [DPF14] for $N \geq 2)$. Let $(u, K)$ be a minimizer. Then there exists $p_{0}>2$ such that $\nabla u \in L_{l o c}^{p_{0}}(\Omega)$.

One of the main ingredient in [DPF14] is, again, the fact that $K$ is nice in many balls to improve how nice $K$ is in reality. In [DPF14] this is phrased in terms of "porosity" of the singular part of $K$, which comes from the Carleson measure behavior of the flatness and normalized energy.

The exponent $p_{0}$ is not explicit. To be honest, the authors of [DPF14] claim in their paper that $p_{0}$ could be made explicit, provided a careful look at their proof but also modifying some needed arguments from the regularity theory, but it is not yet clear at all which kind of value for $p_{0}$ this would produce, probably of type $p_{0}=2+\varepsilon$ with $\varepsilon$ small.

Now the connection between Theorem 3.24 and Theorem 3.22 was actually established much earlier in a paper by Ambrosio, Fusco and Hutchinson [AFH03] (see also [LFR13]) where the following result has been proved.

Theorem 3.25 ([AFH03]). If $\nabla u \in L_{l o c}^{p}(\Omega)$ then

$$
\mathcal{H}-\operatorname{dim}(\Sigma) \leq \max (N-2, N-p / 2) .
$$


Theorem 3.24 together with Theorem 3.25 gives yet another proof for Theorem 3.22 .

In addition in the paper [AFH03], a particular attention has been given to the following particular subset of $\Sigma$,

$$
\Gamma:=\left\{x \in \Sigma \quad ; \quad \lim _{r \rightarrow 0} \frac{1}{r^{N-1}} \int_{B_{r}}|\nabla u|^{2} d x=0\right\} .
$$

It is proved in [AFH03] (see also [LFR13]), that

$$
\mathcal{H}-\operatorname{dim}(\Gamma) \leq N-2,
$$

and this is one of the main ingredient in the proof of Theorem 3.25. Using the regularity theory, we can be a bit more precise than (3.21), and give a local description of points $x \in \Gamma$, at least in dimensions 2 and 3 . Here is an example of statement.

Theorem 3.26. Let $x \in \Gamma$. It follows that:

1. If $N=2$ then $K \cap B(x, r)$ is a nice spider for some $r>0$ (i.e. three $C^{1, \alpha}$ curves meeting by three with 120 degree angles).

2. If $N=3$ then $K \cap B(x, r)$ is either a nice $\mathbb{Y}$ or a nice $\mathbb{T}$ for some $r>0$ (i.e. image of cone of type $\mathbb{Y}$ or $\mathbb{T}$ by a smooth $C^{1, \alpha}$ map).

The proof of 1 . follows from [Dav05b, 60, Proposition 1] and the proof of 2 . is a consequence of [Lem11, Theorem 9].

The issue is then to control the points in $\Sigma \backslash \Gamma$, which is actually related to the so called singular singular set, studied in [Dav05b, Chapter 67] introduced by Léger [Leg99].

Let us finish this section by giving a clear picture in dimensions 2 and 3: it can be shown that $K$ splits in exactly two parts, a first part corresponding to points $x$ for which

$$
\frac{1}{r^{N-1}} \int_{B(x, r)}|\nabla u|^{2} d x \rightarrow 0
$$

and its complement denoted by $K^{\sharp}$ in [Dav05b], the singular singular set, turns out to be exactly the set of $x \in K$ for which

$$
\liminf _{r \rightarrow 0} \frac{1}{r^{N-1}} \int_{B(x, r)}|\nabla u|^{2} d x>\varepsilon_{0},
$$

for some $\varepsilon_{0}>0$ (that does not depend on $x$ ). This dichotomy needs a little proof, but holds true at least in dimensions 2 and 3 .

In dimensions 2 and 3 , the local description for points in $K \backslash K^{\sharp}$ is quite clear, as being points for which $K$ is locally the image of a minimal cone by a smooth map (flat or triple point in dimension 2, $\mathbb{P}$-point, $\mathbb{Y}$-point or $\mathbb{T}$-point in dimension 3 ), whereas about $K^{\sharp}$ we only know up to now that $\mathcal{H}$ - $\operatorname{dim}(\Gamma)<N-1$. 


\section{Global minimizers in dimension 3}

After Theorem 3.3, a natural question is whether one could classify all possible global minimizers in dimension 3. This question is far from being achieved today, whereas some first elements are contained in [Dav05b, H76], [Lem08, Lem09, Lem14] that we will try to describe here.

A first basic question is whether assuming $\nabla u=0$ forces $K$ to be a minimal cone i.e., one of type $\mathbb{P}, \mathbb{Y}$ or $\mathbb{T}$. This was proved in [Dav09] and is exactly the purpose of Theorem 3.14 which could be rephrased in the following way.

Theorem 4.1. [Dav09] If $(u, K)$ is a reduced minimizer in $\mathbb{R}^{3}$ and $\nabla u=0$ then $K$ is either $\emptyset$, or a cone of type $\mathbb{P}, \mathbb{Y}$ or $\mathbb{T}$.

The product of a cracktip times a line also gives a global minimizer in $\mathbb{R}^{3}$, as shown in [Dav05b, H.76]. It is even known that $u$ is necessarily this one whenever $K$ is a half-plane.

Proposition 4.2. [Lem09] The half-plane $K=\mathbb{R}^{-} \times\{0\} \times \mathbb{R}$ is the singular set of a global minimizer in $\mathbb{R}^{3}$, associated to the vertically constant function written in cylindrical coordinates

$$
\left.u(r, \theta, z)=\sqrt{\frac{2}{\pi}} r \sin (\theta / 2), r>0, \theta \in\right]-\pi, \pi[, z \in \mathbb{R} .
$$

Moreover, $K$ being fixed as above, $u$ is the unique possible function that makes $(u, K)$ a global minimizer.

This ends the list of known global minimizers in $\mathbb{R}^{3}$ so far.

\subsection{If $K$ is a cone then $u$ is $1 / 2$-homogeneous}

In order to identify a possible extra global minimizer, it is natural to look at singular set of cone type. This is still an open problem but, assuming so, then it is not very complicated to see that $u$ must be $1 / 2$ homogeneous, up to an additive constant as was shown in [Lem09]. Let us present the proof.

The main ingredients are the standard estimate on Mumford-Shah minimizers

$$
\int_{B_{r}}|\nabla u|^{2} d x \leq C r^{N-1}
$$

obtained by taking $\left(u \mathbf{1}_{B_{r}},\left(K \backslash B_{r}\right) \cup \partial B_{r}\right)$ as a competitor, together with a decomposition of $u$ in spherical harmonics. We recall that the Laplace operator in polar coordinates writes

$$
\Delta=\partial_{r}^{2}+\frac{N-1}{r} \partial_{r}+\frac{1}{r^{2}} \Delta_{S}
$$


where $\Delta_{S}$ stands for the spherical Laplace-Beltrami operator on $\mathbb{S}^{N-1}$. Consequently, if $g$ is an eigenfunction for $-\Delta_{S}$ associated to the eigenvalue $\lambda$, the homogeneous function

$$
h(x)=|x|^{\alpha} g\left(\frac{x}{|x|}\right)
$$

is harmonic provided that

$$
\alpha=\alpha(\lambda)=\frac{\sqrt{(N-2)^{2}+4 \lambda}-(N-2)}{2} .
$$

Now let $K$ be the singular set of a global Mumford-Shah minimizer in $\mathbb{R}^{3}$ associated to some harmonic function $u$. Assume that $K$ is a cone, and also that it is regular enough so that the embedding $W^{1,2}\left(\mathbb{S}^{N-1} \backslash K\right) \rightarrow L^{2}\left(\mathbb{S}^{N-1}\right)$ is compact. Then there is a basis of $L^{2}\left(\mathbb{S}^{N-1}\right)$ formed by eigenfuntions of the Neumann-Laplace-Beltrami operator on $\mathbb{S}^{N-1}$, that we denote by $\left\{g_{k}\right\}_{k} \geq 0$. Writing the trace of $u$ in this basis yields

$$
\left.u\right|_{\mathbb{S}^{N-1}}=\sum_{k \geq 0} a_{k} g_{k}
$$

for some coefficients $a_{k}$. Then considering the function

$$
w(x)=\sum_{k \geq 0} a_{k}|x|^{\alpha_{k}} g_{k}\left(\frac{x}{|x|}\right)
$$

with the right powers $\alpha_{k}$, and assuming that it converges properly, we notice that $w$ is a harmonic function that satisfies a Neumann boundary condition on $K \cap B_{1}$ and that coincide with $u$ on $\partial B_{1} \backslash K$. By uniqueness we must have $u=w$. It is also not difficult to check that what was just done in $B_{1}$ can actually be done in any $B_{R}$ with the same coefficients, thus the writing

$$
u(x)=\sum_{k \geq 0} a_{k}|x|^{\alpha_{k}} g_{k}\left(\frac{x}{|x|}\right)
$$

holds true in the whole $\mathbb{R}^{N} \backslash K$. One can also check that the $|x|^{\alpha_{k}} g_{k}\left(\frac{x}{|x|}\right)$ are actually orthogonal in $W^{1,2}\left(B_{R}\right)$ for all $R$.

After checking all this, we can use the estimate (4.2), the orthogonality of the terms in the sum, and then pass to the limit when $r \rightarrow 0$ and $r \rightarrow+\infty$. This implies that all the terms in the sum must be zero, except the ones which behave exactly like the estimate (4.2), namely we must have $\alpha=1 / 2$. Let us summarize in the following statement.

Proposition 4.3. [Lem09] If $(u, K)$ is a global minimizer in $\mathbb{R}^{N}$, and if $K$ is a (smooth enough) cone, then up to adding a locally constant function, $u$ has the form $u=C|x|^{1 / 2} g\left(\frac{x}{|x|}\right)$, where $C \in \mathbb{R}$. In particular its restriction on the spherical domain $\mathbb{S}^{N-1}$ is an eigenfunction for the spherical Neumann-Laplace-Beltrami operator in $\mathbb{S}^{N-1} \backslash K$ associated to the eigenvalue $(2 N-3) / 4$. 
The above proposition can be used to eliminate some possible candidates, when we know the exact value for the associated eigenvalue. We mention below some of them.

Corollary 4.4. Let $(u, K)$ be a global minimizer in $\mathbb{R}^{3}$. Then

1. K cannot be an angular sector different from a plane or a half-plane.

2. K cannot be a wing (two half planes meeting along their edges making an angle $\theta)$ different from a plane or a half-plane.

3. If $K$ meets the sphere in such a way that its complement is a union of convex domains, each of them being contained in a hemisphere, then $K$ is a cone of type $\mathbb{P}, \mathbb{Y}$ or $\mathbb{T}$.

The proof of 1. and 2. can be found in [Lem09], it directly comes from estimates of the eigenvalue obtained in [Dau92]. For the last point 3. it was never stated before, but it directly comes from the eigenvalue estimate contained in [Maz09], that was not known by the author at the time when [Lem09] was written.

In [Dav05b], the construction of a possible extra minimizer is suggested as follows. Let $\Omega$ be a cylinder in $\mathbb{R}^{3}$ based on the unit 2 -d ball, of height 1 , say. Let then $g$ be a cylindrical function that would start with a singularity of type $\mathbb{Y}$ at the level $z=0$ and end with no singularity at all at $z=1$ (take for instance a locally constant function in $\mathbb{R}^{3}$ that would jump across a cone of type $\mathbb{Y}$, and multiply it by cut-off function in the $z$ direction that goes from 1 at $z=0$, to 0 at $z=1 / 2$ ). Now minimize the Mumford-Shah functional with this function $g$. And finally take the blow-up limit at the origin of that minimizer. What should it be ? It was conjectured by David the existence of a new global minimizer that should look like a cone $\mathbb{Y}$ bevelled, whose intersection with the sphere would look like the union of three branches of arc of circles of length to be determined, meeting by 120 degree. But this possibility has been, surprisingly, excluded by Merlet in [Mer07] by use of a numerical method.

The rest of this section is devoted to giving a partial answer to the Open Problem 3.20 .

\subsection{A monotonicity formula in higher dimensions}

In order to classify the possible global minimizers in higher dimension, it would be very useful to have at hand a monotonicity formula as the one of Bonnet in dimension 2. Unfortunately, a similar monotonicity is not known to hold in general. However, in the very particular case when $K$ is contained in a smooth enough cone, one can derive some monotonicity for a suitable normalized energy as proved in [Lem14], which leeds to the rigidity result of the next section. 
Definition 4.5. If $N \geq 3$, a closed set $K \subset \mathbb{R}^{N}$ will be called a Neumann cone if the three following properties hold:

1. $K$ is a cone.

2. $K \cap \mathbb{S}^{N-1}$ is $(N-2)$-rectifiable and $\mathcal{H}^{N-2}\left(K \cap \mathbb{S}^{N-1}\right)<+\infty$.

3. The embedding $W^{1,2}\left(\mathbb{S}^{N-1} \backslash K\right) \rightarrow L^{2}\left(\mathbb{S}^{N-1}\right)$ is compact.

It is easy to check that a hyperplane in $\mathbb{R}^{N}$ containing the origin is a Neumann cone, or that a half-hyperplane is a Neumann cone. Moreover, when $K$ is a Neumann cone then the first positive eigenvalue of the Neumann Laplacian, denoted by $\lambda_{1}\left(\mathbb{S}^{2} \backslash\right.$ $K)$, is well defined. This is also the case for any set $K$ contained in a Neumann cone, because then the embedding $W^{1,2}\left(\mathbb{S}^{N-1} \backslash K\right) \rightarrow L^{2}\left(\mathbb{S}^{N-1}\right)$ remains compact.

Now here is an example of statement for the monotonicity result (see [Lem14] for the general case where assumption 2. of Lemma 4.6 is replaced by a more general topological assumption). We call energy minimizer in $\mathbb{R}^{N} \backslash K$, a function $u$ that locally minimizes $\int_{\mathbb{R}^{N} \backslash K}|\nabla u|^{2} d x$ in $\mathbb{R}^{N} \backslash K$. This function is harmonic in $\mathbb{R}^{N} \backslash K$ with a Neumann boundary condition on $K$, i.e. zero normal derivative on $K$, in a weak sense.

Lemma 4.6 (Monotonicity in higher dimension). [Lem14] Let $K \subset \mathbb{R}^{N}$ be a closed set and $\gamma>0$ satisfy the following assumptions.

1. $K$ is contained in a Neumann cone.

2. $\partial B_{r} \backslash K$ is connected for a.e. $r>0$.

3. For a.e. $r>0$, the first positive eigenvalue of the Neumann-Laplace-Beltrami operator on $\partial B_{r} \backslash K$ satisfies $\lambda_{1}\left(\partial B_{r} \backslash K\right) \geq \gamma / r^{2}$.

Then for every local energy minimizer $u$ in $\mathbb{R}^{N} \backslash K$ we have that

$$
\varphi: r \mapsto \frac{1}{r^{\alpha}} \int_{B_{r}} \frac{|\nabla u|^{2}}{|x|^{N-2}} d x
$$

is nondecreasing, where $\alpha$ is defined through

$$
\alpha=\alpha(N, \gamma)=\sqrt{(N-2)^{2}+4 \gamma}-(N-2) .
$$

Moreover, if $\varphi(r)$ is a nonzero constant on an interval $[a, b]$ then for a.e. $r \in[a, b]$ the value $\gamma / r^{2}$ is the first positive eigenvalue for the Neumann-Laplace-Beltrami operator on $\partial B_{r} \backslash K$ and the restriction of $u$ to $\partial B_{r} \backslash K$ is an associated eigenfunction. 
Notice that the energy is no more the Dirichlet integral but a weight $1 /|x|^{N-2}$ has been added; this was inspired by the famous Alt-Caffarelli-Friedman monotonicity formula [ACF84]. One can indeed try as an exercice to proceed the same way as Bonnet for $\int_{B_{r}}|\nabla u|^{2} d x$ in dimension 3 with a homogeneous fonction in the complement of a cone, and see that it cannot work. Hence the right energy to consider in higher dimension is probably the one in (4.3).

Elements of proof for Lemma 4.6. The proof looks like the one of Bonnet, and relies on an integration by parts. Before that we regularize the norm by setting

$$
|x|_{\varepsilon}=\sqrt{x_{1}^{2}+\cdots+x_{N}^{2}+\varepsilon}
$$

and we notice that, the function $h_{\varepsilon}:=|x|_{\varepsilon}^{2-N}$ verifies $\Delta h_{\varepsilon} \leq 0$.

We may assume without loss of generality that the average of $u$ on $\mathbb{S}^{N-1}$ is zero. Since $\Delta\left(u^{2}\right)=2|\nabla u|^{2}$ by the Gauss-Green formula (which can be rigorously justified, see [Lem14]) applied in $B_{r} \backslash K$ we infer that

$$
\begin{aligned}
& 2 \int_{B_{r} \backslash K} \frac{|\nabla u|^{2}}{|x|_{\varepsilon}^{N-2}} d x=\int_{B_{r} \backslash K} h_{\varepsilon} \Delta\left(u^{2}\right) d x \\
\leq & \int_{\partial B_{r} \backslash K} h_{\varepsilon} 2 u \frac{\partial u}{\partial \nu}-\int_{\partial B_{r} \backslash K} u^{2} \frac{\partial h_{\varepsilon}}{\partial \nu}+\int_{K \cap B_{r}}\left(\left(u^{2}\right)^{+}-\left(u^{2}\right)^{-}\right) \frac{\partial h_{\varepsilon}}{\partial \nu} .
\end{aligned}
$$

We forgot the term $\int_{B_{r} \cap K} h_{\varepsilon} 2 u \frac{\partial u}{\partial \nu}$ because $\frac{\partial u}{\partial \nu}=0$ on $K$, and we also forgot the terms of the form $\int_{B_{r} \backslash K} u^{2} \Delta h_{\varepsilon}$ because they are negative (which explains the inequality). Finally, by $\left(u^{2}\right)^{+}$and $\left(u^{2}\right)^{-}$we intend the traces of $u^{2}$ on both sides of $K$. This traces exists $\mathcal{H}^{N-1}$-a.e. and can be defined using the theory of $S B V$ functions.

Letting $\varepsilon$ tends to zero we get

$$
\leq 2 r^{2-N} \int_{\partial B_{r} \backslash K} u \frac{\partial u}{\partial \nu}+\frac{N-2}{r^{N-1}} \int_{\partial B_{r} \backslash K} u^{2}+(N-2) \int_{B_{r} \cap K}\left(\left(u^{2}\right)^{+}-\left(u^{2}\right)^{-}\right) \frac{x}{|x|^{N}} \cdot \nu .
$$

Now the key point is that, because $K$ is contained in a cone, then the normal $\nu$ to $K$ at $x$ is orthogonal to the vector $x$, in other words $x \cdot \nu=0$ on $K$ and the last integral term in the above expression is zero, yielding,

$$
\int_{B_{r} \backslash K} \frac{|\nabla u|^{2}}{|x|^{N-2}} d x \leq r^{2-N} \int_{\partial B_{r} \backslash K} u \frac{\partial u}{\partial \nu} d \omega+\frac{N-2}{2 r^{N-1}} \int_{\partial B_{r} \backslash K} u^{2} d \omega .
$$

We then use Cauchy-Schwarz and the elementary inequality $a b \leq \frac{1}{2 \delta} a^{2}+\frac{\delta}{2} b^{2}$ to 
write, setting $U=\partial B_{r} \backslash K$,

$$
\begin{aligned}
& \int_{B_{r} \backslash K} \frac{|\nabla u|^{2}}{|x|^{N-2}} \leq r^{2-N} \int_{U} u \frac{\partial u}{\partial \nu} d \mathcal{H}^{N-1}+\frac{N-2}{2 r^{N-1}} \int_{U} u^{2} d \mathcal{H}^{N-1} \\
\leq & r^{2-N}\left(\int_{U} u^{2}\right)^{\frac{1}{2}}\left(\int_{U}\left(\frac{\partial u}{\partial \nu}\right)^{2}\right)^{\frac{1}{2}}+\frac{N-2}{2 r^{N-1}} \int_{U} u^{2} \\
\leq & r^{2-N} \frac{1}{\sqrt{\lambda_{1}(U)}}\left(\int_{U}\left|\nabla_{\tau} u\right|^{2}\right)^{\frac{1}{2}}\left(\int_{U}\left(\frac{\partial u}{\partial \nu}\right)^{2}\right)^{\frac{1}{2}}+\frac{(N-2)}{2 r^{N-1} \lambda_{1}(U)} \int_{U}\left|\nabla_{\tau} u\right|^{2} \\
\leq & r^{2-N} \frac{r}{\sqrt{\gamma}}\left(\int_{U}\left|\nabla_{\tau} u\right|^{2}\right)^{\frac{1}{2}}\left(\int_{U}\left(\frac{\partial u}{\partial \nu}\right)^{2}\right)^{\frac{1}{2}}+\frac{(N-2) r^{2}}{2 r^{N-1} \gamma} \int_{U}\left|\nabla_{\tau} u\right|^{2} \\
\leq & \frac{r^{3-N}}{\sqrt{\gamma}}\left(\frac{\delta}{2} \int_{U}\left|\nabla_{\tau} u\right|^{2}+\frac{1}{2 \delta} \int_{U}\left(\frac{\partial u}{\partial \nu}\right)^{2}\right)+\frac{(N-2) r^{3-N}}{2 \gamma} \int_{U}\left|\nabla_{\tau} u\right|^{2} \\
\leq & r^{3-N}\left(\frac{\delta}{2 \sqrt{\gamma}}+\frac{(N-2)}{2 \gamma}\right) \int_{U}\left|\nabla_{\tau} u\right|^{2}+\frac{r^{3-N}}{2 \delta \sqrt{\gamma}} \int_{U}\left(\frac{\partial u}{\partial \nu}\right)^{2} .
\end{aligned}
$$

Then we choose $\delta>0$ so that

$$
\left(\frac{\delta}{2 \sqrt{\gamma}}+\frac{(N-2)}{2 \gamma}\right)=\frac{1}{2 \delta \sqrt{\gamma}}
$$

This gives the value

$$
\delta=\frac{\sqrt{(N-2)^{2}+4 \gamma}-(N-2)}{2 \sqrt{\gamma}}
$$

which implies the inequality,

$$
\int_{B_{r} \backslash K} \frac{|\nabla u|^{2}}{|x|^{N-2}} \leq \frac{1}{\alpha} r^{3-N} \int_{\partial B_{r} \backslash K}|\nabla u|^{2},
$$

with the $\alpha$ defined in the statement of the Lemma. But this exactly says that

$$
E(r) \leq \frac{1}{\alpha} r E^{\prime}(r)
$$

with

$$
E(r)=\int_{B_{r} \backslash K} \frac{|\nabla u|^{2}}{|x|^{N-2}},
$$

in other words $\frac{d}{d r}\left(r^{-\alpha} E(r)\right) \geq 0$ and $r^{-\alpha} E(r)$ is nondecreasing.

Finally to finish the proof, we observe that when $\varphi$ is constant, the derivative is zero, in other words we must have $E(r)=\frac{1}{\alpha} r E^{\prime}(r)$ for a.e. $r>0$, thus, all the inequalities that we used to prove the monotonicity (starting from the second line) must be equalities. In particular the equality in the third line says that the restriction of $u$ to almost every sphere must be the optimal function in the Poincaré-Wirtinger inequality associated with the constant $r^{2} / \gamma$, and so the Lemma follows. 


\subsection{A rigidity result for $K$ contained in a half-plane}

The purpose of this section is to present the proof of the following result.

Theorem 4.7. [Lem14] Let $(u, K)$ be a (reduced) global minimizer in $\mathbb{R}^{3}$ with $K$ contained in a half plane, and touching its edge. Then $K$ is the half-plane.

Elements of proof. Without loss of generality, we can assume that the origin is situated on the edge of the half-plane, and contained in $K$. It is known that the first eigenvalue of a sphere minus a half-equator is equal to $3 / 4$. By the monotonicty property of the Neumann eigenvalues with respect to removing a negligible set, we are exactly under the hypothesis of Lemma 4.6 with $\gamma=3 / 4$ because $K$ is contained in a half plane. Therefore

$$
\varphi(r):=\frac{1}{r} \int_{B_{r} \backslash K} \frac{|\nabla u|^{2}}{|x|}
$$

is nondecreasing, and the limit at 0 and $+\infty$ exists. Let us denote them respectively by $f_{0}$ and $f_{\infty}$. We claim that $f_{0}$ and $f_{\infty}$ are finite. To check this we shall use the elementary inequality (4.2). Notice that the constant is only dimensional: $C=\omega_{N}$ is the measure of the $N$-1-dimensional unit sphere. Now using Fubini and Chebychev, for any $r>0$ we can choose $r_{0} \in(r, 2 r)$ such that

$$
\int_{\partial B_{r_{0}} \backslash K}|\nabla u|^{2} d \omega \leq \frac{1}{r} \int_{\left(B_{2 r} \backslash B_{r}\right) \backslash K}|\nabla u|^{2} d x \leq 4 r \omega_{3}
$$

and such that (4.5) holds, from which we deduce that

$$
\int_{B_{r} \backslash K} \frac{|\nabla u|^{2}}{|x|} \leq \int_{B_{r_{0}} \backslash K} \frac{|\nabla u|^{2}}{|x|} \leq r_{0} \int_{\partial B_{r_{0}} \backslash K}|\nabla u|^{2} \leq 4 \omega_{3} r,
$$

This implies that $\varphi(r)$ is uniformly bounded for $r>0$ thus $f_{0}$ and $f_{\infty}$ are finite, and we readily have that $f_{0} \leq f_{\infty}<+\infty$.

Let us check moreover that $f_{0}>0$. Indeed if not, then one would have, for $r>0$ small enough,

$$
\frac{1}{r^{2}} \int_{B_{r} \backslash K}|\nabla u|^{2} \leq \frac{1}{r} \int_{B_{r} \backslash K} \frac{|\nabla u|^{2}}{|x|} \leq \varepsilon_{0},
$$

where $\varepsilon_{0}$ is the same as the statement of Theorem 3.11. But then $K \cap B_{c r}$ must be the image of a minimal cone by a $C^{1}$-smooth map, containing 0 in its interior. This is a contradiction with the fact that the origin lies on the edge of the half-plane containing $K$. We therefore conclude that $f_{0}>0$.

Now we take blow-up and blow-in limits. We begin with the blow-up. Let $u_{k}:=$ $\frac{1}{\sqrt{r_{k}}} u\left(r_{k} x\right)$ and $K_{k}:=\frac{1}{r_{k}} K$ with any $r_{k} \rightarrow 0$. Then, by Theorem 2.8, up to a subsequence (not relabeled), the sequence $\left(u_{k}, K_{k}\right)$ converges to some $\left(u_{0}, K_{0}\right)$ (in 
the sense of Definition 2.7), and $\left(u_{0}, K_{0}\right)$ is still a reduced global minimizer, with $K_{0}$ still satisfying assumptions 1,2 , and 3 of Theorem 4.6. Moreover for any $R>0$ and $k \in \mathbb{N}$ we have that

$$
\frac{1}{R} \int_{B_{R}} \frac{\left|\nabla u_{k}\right|^{2}}{|x|} d x=\frac{1}{r_{k} R} \int_{B_{r_{k} R}} \frac{|\nabla u|^{2}}{|x|} d x \underset{k \rightarrow+\infty}{\longrightarrow} f_{0} .
$$

On the other hand, we know that $\nabla u_{k}$ converges to $\nabla u_{0}$ in $L^{2}\left(B_{M}\right)$ for any $M>$ 0 . It is actually not very difficult, using the estimate (4.6) from the proof of the Monotonicity lemma, that the following stronger convergence holds true

$$
\int_{B_{R}} \frac{\left|\nabla u_{k}\right|^{2}}{|x|} d x \underset{k \rightarrow+\infty}{\longrightarrow} \int_{B_{R}} \frac{\left|\nabla u_{0}\right|^{2}}{|x|} d x .
$$

Returning now to (4.7), we obtain that

$$
\frac{1}{R} \int_{B_{R}} \frac{\left|\nabla u_{0}\right|^{2}}{|x|} d x=f_{0}, \quad \forall R>0 .
$$

But, since $f_{0} \neq 0$, the last conclusion of Lemma 4.6 then says that $\lambda_{1}\left(\partial B_{R} \backslash K_{0}\right)=$ $3 / 4$ for a.e $R>0$, and it follows that $K_{0}$ is a half-plane, and therefore $u_{0}$ is Cracktip $\times \mathbb{R}$ up to some additive constant.

Then we do exactly the same for $u_{\infty}$ and conclude that, akin to the blow-up limit, the normalized energy associated to the blow-down limit must be constant as well. Thus up to an additive constant, $u_{\infty}$ is the same function $\sqrt{2 r / \pi} \sin (\theta / 2)$ as $u_{0}$ (with the same constant in front), and therefore $f_{0}=f_{\infty}$. But then returning to the function $u$ and in virtue of the monotonicity of $\varphi(r)$, we deduce that $\varphi(r)$ is constant on $(0,+\infty)$, and consequently $K$ must be a half-plane itself.

\section{Some links with classical mechanics}

The Mumford-Shah functional, or some variants, is also used in several models from classical mechanics. The purpose of what follows is to mention some of them.

\subsection{The crack model of Francfort and Marigo}

\subsubsection{Introduction of the model}

According to Griffith's theory, the propagation of a brittle fracture in an elastic body is governed by the competition between the energy spent to produce a crack, proportional to its length, and the corresponding release of bulk energy. An energetic formulation of this idea is the core of variational models for crack propagation, which 
were introduced by Francfort and Marigo in [FM98] and are based on a MumfordShah-type functional.

If $\Omega \subset \mathbb{R}^{N}$ (usually $N=3$, sometimes $N=2$ for simplicity) is the reference configuration of an elastic body subject to a displacement $u: \Omega \rightarrow \mathbb{R}^{N}$ with prescribed boundary datum $u=g$ on $\partial \Omega$, the elastic energy is given by

$$
\frac{1}{2} \int_{\Omega} \mathbf{A} e(u): e(u) d x
$$

where $e(u)=\frac{1}{2}\left(D u+D u^{T}\right)$ is the symmetrical part of the gradient of $u$, the notation ": " denotes the usual scalar product on matrices, and A is the fourth order Hooke's tensor

$$
\mathbf{A} e=\lambda \operatorname{Tr}(e) I d+2 \mu e .
$$

The constants $\lambda>0$ and $\mu>0$ are the so-called Lamé coefficients, and minimizers of the "Dirichlet type" energy (5.1) are solutions to an elliptic system called the Lamé system. For a given crack $K \subset \Omega$, the value of

$$
E(K, g):=\min _{u \in L D(\Omega \backslash K) ; u=g \text { on } \partial \Omega} \frac{1}{2} \int_{\Omega} \mathbf{A} e(u): e(u) d x,
$$

is called the bulk energy (the space $L D$ being those of $u \in L^{2}$ with $e(u)$ in $L^{2}$ ).

There is a particular case, called "anti-plane shear", where the energy (5.1) reduces to the classical Dirichlet energy. This happens when the domain is a cylinder $\Omega \times \mathbb{R}$, with $\Omega \subset \mathbb{R}^{2}$, and assuming the crack to be vertically invariant, while the displacement is vertical only. Under those assumptions, the problem reduces to a purely 2D scalar problem, and the energy involved reduces to the classical MumfordShah energy. It is often useful to reduce to this simpler case for which the tools from the Mumford-Shah functional can directly apply.

But one of the main difference with the original Mumford-Shah problem, is that the growth of a crack in an elastic body is an evolution process.

For simplicity we now restrict ourselves exclusively to the case $N=2$. The idea of Francfort and Marigo is to consider, for a given time-dependent loading process $g(t)$ on $\partial \Omega$, the quasi-static evolution of the Mumford-Shah type energy

$$
\frac{1}{2} \int_{\Omega \backslash K} \mathbf{A} e(u): e(u) d x+\kappa \mathcal{H}^{1}(K)
$$

where the constant $\kappa>0$ is related to the toughness of the material.

The functional (5.3) looks like a simple variant of the standard Mumford-Shah functional, but it is just a foggy analogy since most of the desired regularity results are still unknown. Not even the starting point of the regularity theory, that is, the density lower boun. In other words there is no analogue for this functional, to the famous De Giorgi-Carriero-Leaci paper [DGCL89]. Any $C^{1}$ regularity result would be also welcome but this looks out of reach for the moment. 
Open problem 5.1. Prove that any minimizer for the functional (5.3) is Ahlforsregular.

Now, the construction of the evolution proceeds as follows: first discretize the time line via $0<t_{1}<\cdots<t_{k}<t_{k_{0}}$. Then construct $\left(u_{k}, K_{k}\right)$ by induction. If the pair is already constructed at time $k$, then $\left(u_{k+1}, K_{k+1}\right)$ is the solution for the problem

$$
\min _{(u, K) ; K \supseteq K_{k} ; u=g\left(t_{k+1}\right) \text { on } \partial \Omega}\left\{\frac{1}{2} \int_{\Omega \backslash K} \mathbf{A} e(u): e(u) d x+\kappa \mathcal{H}^{1}(K)\right\} .
$$

Then let $\max _{k}\left|t_{k}-t_{k+1}\right|$ tend to zero and pass to the limit. This should give a time dependent pair $(u(t), K(t))$ which satisfies Griffith's criterium for the evolution of a brittle fracture, which in turn, reduces to write the optimality conditions related to this variational construction. The first mathematical construction in that direction was obtained by Dal Maso and Toader [DMT02] in the simple 2D linearized antiplane setting, then extended in various directions by other authors [Cha03, DMFT05, FL03, BG14].

But even if the real true object to study is the evolution of $K(t)$ depending on time $t$, some interesting questions already arise at a freezed time $t_{0}$, for which the technics and tools from the original Mumford-Shah problem could be quite useful. A general question of that type is the following : let $K\left(t_{0}\right)$ be a pre-crack at time $t_{0}$. How would the crack path grow during the evolution? Is it continuous in time ? Where it will appear ? And what direction will be privileged ?

Examples of physical quantities related to those questions are the so-called stress intensity factor and energy release rate, which were the central subject of the papers [CL13, BCL15] and that we present below.

Let us finally mention that other various modifications of the functional like for instance changing $\mathcal{H}^{1}(K)$ with $\int_{K} f\left(\left|u^{+}-u^{-}\right|\right) d \mathcal{H}^{1}$ for some suitable function $f$ with appropriate profile are quite useful in other models from elasticity theory, especially for plastic material. Also, some "phase-field" approximation of the functional first introduced in [AT92] was used later in damage models, see for instance [Iur13].

\subsubsection{Stress intensity factor and Energy release rate}

In the quasistatic model, the fracture $K(t)$ is in equilibrium at any time, which means that the total energy cannot be improved at time $t_{0}$ by extending the crack. Precisely, for any closed sets $K \supseteq K\left(t_{0}\right)$ such that $K\left(t_{0}\right) \cup K$ is connected, and for any $u$ satisfying $u=g\left(t_{0}\right)$ on $\partial \Omega \backslash\left(K\left(t_{0}\right) \cup K\right)$, one must have that

$$
E\left(K\left(t_{0}\right), g\left(t_{0}\right)\right)+\kappa \mathcal{H}\left(K\left(t_{0}\right)\right) \leq \int_{\Omega \backslash\left(K\left(t_{0}\right) \cup K\right)} \mathbf{A} e(u): e(u) d x+\kappa \mathcal{H}(K) .
$$


This implies that the propagation of the crack is totally dependent on the external force $g$, and a necessary condition for $K$ to propagate is that of the first order limit of the bulk energy, namely

$$
\limsup _{h \rightarrow 0^{+}} \frac{E\left(K\left(t_{0}+h\right), g\left(t_{0}+h\right)\right)-E\left(K\left(t_{0}\right), g\left(t_{0}\right)\right)}{h},
$$

to be greater or equal to $\kappa$. The limit in (5.5) can be interpreted as an energy release rate along the growing crack, which is the central object of many recent works [CGP08, CFM10, CFM09, KKT10, LT11]. In particular, one can see the limit in (5.5) as a $\Gamma$-limit, since it is a limit of minimal values.

Another useful quantity is the so-called stress intensity factor: if $K$ is a half-line and $u$ is minimizing the bulk energy in the scalar case, then $u$ is a harmonic function (or more generally satisfies an elliptic equation) with Neumann boundary condition on $K$ thus can be written as a sum of terms composed by homogeneous harmonic functions, and the first non constant one is the famous $C \sqrt{r} \sin (\theta / 2)$. Now if $K$ is no more a half-line but asymptotically converging to a half-line at small scales, then the suitable renormalized blow-up of $u$ should converge to a function of type $C \sqrt{r} \sin (\theta / 2)$. The constant $C$ in front is called the stress intensity factor. It is related to the quantity

$$
\lim _{r \rightarrow 0} \frac{1}{r} \int_{B_{r} \backslash K}|\nabla u|^{2} d x
$$

provided that the limit exists, and in some sense quantifies the winning in terms of elastic energy by adding an infinitesimally small piece of crack at the tip. The existence of the limit in (5.6) follows in general by the monotonicity argument of Bonnet [Bon96] seen before. This is the first step to prove the convergence of the blow-up limit of $u$ to the function $C \sqrt{r} \sin (\theta / 2)$, when $K$ is merely closed, connected with density $1 / 2$ at the tip (which implies a convergence to a half-line, up to a possible sequence of rotations). This was done in the paper [CL13].

A similar work has been done in the vectorial case in [BCL15], but the major difference is that no monotonicity is known for the normalized energy. Consequently, everything is proved "up to a subsequence" and the existence of a true limit is not known, which leads to the following open question (a similar question about scalar bi-harmonic functions could be stated via a duality argument like in [BCL15]).

Open problem 5.2. Prove a monotonicity result of Bonnet type for minimizers of (5.1). More precisely, assume that $K \subset \Omega$ is compact connected, that $u: \Omega \backslash K \rightarrow \mathbb{R}^{2}$ is a local energy minimizer, in other words satisfies

$$
\int_{B \backslash K}|e(u)|^{2} d x \leq \int_{B \backslash K}|e(v)|^{2} d x,
$$


for all $B$ compactly contained in $\Omega$ and for all $v$ that are equal to $u$ on $B^{c}$. Then is it true that

$$
\varphi: r \mapsto \frac{1}{r} \int_{B_{r} \backslash K}|e(u)|^{2} d x
$$

is nondecreasing? or more generally, does the limit $\lim _{r \rightarrow 0} \varphi(r)$ always exists?

Anyway up to subsequences, the energy release rate can be associated with a minimizing problem in the whole plane. This has been proved in [CFM10] assuming that $K$ is exactly a straight segment near the origin, while in [BCL15] it has been established for any compact connected sets with density $1 / 2$ at the origin.

We denote by $\mathcal{K}(\bar{\Omega})$ the set of all compact connected subsets of $\Omega$.

Theorem 5.3. Let $K \in \mathcal{K}(\bar{\Omega})$ be a pre-crack with density $1 / 2$ at the origin (i.e. $\frac{1}{r} \mathcal{H}^{1}\left(K \cap B_{r}(0)\right) \rightarrow_{r \rightarrow 0}$ 1) and let $\left(\Gamma_{\varepsilon}\right)_{\varepsilon>0}$ be a sequence of crack increments in $\mathcal{K}(\bar{\Omega})$ be such that $\sup _{\varepsilon} \mathcal{H}^{1}\left(\Gamma_{\varepsilon}\right)<\infty$, and $\Gamma_{\varepsilon} \rightarrow \Gamma$ in the sense of Hausdorff in $\bar{\Omega}$. Remembering the definition of $E(K, g)$ in (5.2), we denote by $u_{K}$ the minimizer of $E(K, g)$. We also denote by $\Sigma_{0}$ the left half $x$-axis and by $u_{\Sigma_{0}}$ the $1 / 2$-homogeneous function that arises as a blow-up limit for $u_{K}$, up to subsequence and rotations. This limit indeed exists. Precisely, for every sequence $\left(\varepsilon_{n}\right) \searrow 0^{+}$, there exist a subsequence $\left(\varepsilon_{k}\right) \equiv\left(\varepsilon_{n_{k}}\right) \searrow 0^{+}$and a rotation $\mathcal{R} \in S O(2)$ such that along which $u_{j}-\bar{u}_{j}$ converges to $u(0)+u_{\Sigma_{0}}$, and

$$
\lim _{k \rightarrow \infty} \frac{1}{\varepsilon_{k}}\left(E\left(K \cup \varepsilon_{k} \Gamma_{\varepsilon_{k}}, g\right)-E(K, g)\right)=\mathcal{F}(\Gamma)
$$

where $\mathcal{F}$ is defined by

$$
\begin{aligned}
\mathcal{F}(\Gamma):=\min _{w \in L D\left(\mathbb{R}^{2} \backslash\left(\Sigma_{0} \cup \mathcal{R}(\Gamma)\right)\right)}\left\{\frac{1}{2} \int_{\mathbb{R}^{2}} \mathbf{A} e(w): e(w) d x\right. & +\int_{B_{R}} \mathbf{A} e\left(u_{\Sigma_{0}}\right): e(w) d x \\
& \left.-\int_{\partial B_{R}} \mathbf{A} e\left(u_{\Sigma_{0}}\right):(w \odot \nu) d \mathcal{H}^{1}\right\},
\end{aligned}
$$

where $R>0$ is any radius such that $\Gamma \subset B_{R}$.

Notice that using an integration by parts, one can show that the expression appearing in (5.8) is actually independent of $R>0$.

\subsubsection{Maximum release of energy for a straight segment}

A natural question is the study of maximizers for the energy release rate, in other words minimizers of (5.8), among all $\Gamma$ satisfying $\mathcal{H}^{1}\left(\Gamma \backslash \Sigma_{0}\right) \leq \ell$. The aim of this section is to show how the knowledge about classical Mumford-Shah minimizers can 
answer this question, in the scalar case. The following proposition, coming from an idea due to Guy David, has never been published before.

Let us restrict ourself to the scalar case (anti-plane). Then the functional $\mathcal{F}$ reduces to

$$
\begin{aligned}
\mathcal{J}(\Gamma):=\min _{u \in H^{1}\left(\mathbb{R}^{2} \backslash\left(\Sigma_{0} \cup \Gamma\right)\right)}\left\{\frac{1}{2} \int_{\mathbb{R}^{2}}|\nabla u|^{2} d x+\int_{B_{R}}\left\langle\nabla\left(u_{\Sigma_{0}}\right), \nabla u\right\rangle d x\right. & \\
& \left.-\int_{\partial B_{R}} u_{\Sigma_{0}} \frac{\partial u}{\partial \nu} d \mathcal{H}^{1}\right\} .
\end{aligned}
$$

Notice that using the direct method of calculus of variation, the problem (5.9) admits a minimizer. Moreover, let us check here that the expression in the definition of $\mathcal{J}(\Gamma)$ does not depend on $R>0$. Indeed, if $R^{\prime}>R$ then

$$
\begin{aligned}
& \int_{B_{R^{\prime}}}\left\langle\nabla\left(u_{\Sigma_{0}}\right), \nabla u\right\rangle d x-\int_{\partial B_{R^{\prime}}} u_{\Sigma_{0}} \frac{\partial u}{\partial \nu} d \mathcal{H}^{1} \\
= & \int_{B_{R}}\left\langle\nabla\left(u_{\Sigma_{0}}\right), \nabla u\right\rangle d x+\int_{B_{R^{\prime}} \backslash B_{R}}\left\langle\nabla\left(u_{\Sigma_{0}}\right), \nabla u\right\rangle d x-\int_{\partial B_{R^{\prime}}} u_{\Sigma_{0}} \frac{\partial u}{\partial \nu} d \mathcal{H}^{1} \\
= & \int_{B_{R}}\left\langle\nabla\left(u_{\Sigma_{0}}\right), \nabla u\right\rangle d x-\int_{\partial B_{R}} u_{\Sigma_{0}} \frac{\partial u}{\partial \nu} d \mathcal{H}^{1} .
\end{aligned}
$$

The aim of this section is to prove the following.

Proposition 5.4. In the scalar case (anti-plane) one has

$$
\mathcal{J}([0, \ell] \times\{0\})=\min _{\Gamma ; \mathcal{H}^{1}\left(\Gamma \backslash \Sigma_{0}\right) \leq \ell} \mathcal{J}(\Gamma) .
$$

If we return to the crack propagation model, Proposition 5.4 means that, if a pre-existing crack at time $t_{0}$ has, say, a left-tangent at the tip of the crack, then the forthcoming crack may want to grow by following the same direction as the half-tangent because it has a maximum release of energy in that direction $(\mathcal{J}$ is a negative quantity). Unfortunately, our result does not say that this is the only possible direction, since the minimizer may not be unique. This is actually an interesting open question, related to what physicists call the "kinking" of a crack. It is not well understood whether or why a crack path may want to "kink" (i.e. change direction suddenly) at certain times.

The proof of the proposition relies on the fact that the cracktip function is a global Mumford-Shah minimizer [BD01]. To do so, we first interpret the problem (5.9) from another point of view.

We still denote by $\Sigma_{0}$ the left half-axis and by $u_{\Sigma_{0}}=\sqrt{\frac{2}{\pi} r} \sin (\theta / 2)$ the cracktip function. For every $R>0$ and every $K \subset B(0, R) \subset \mathbb{R}^{2}$ compact, connected 
containing the origin, we denote by $u_{K, R}$ the solution of

$$
\min _{w \in H^{1}\left(B_{R} \backslash\left(\Sigma_{0} \cup K\right)\right)}\left\{\int_{B_{R} \backslash\left(\Sigma_{0} \cup K\right)}|\nabla w|^{2} d x ; w=u_{\Sigma_{0}} \text { on } \partial B_{R} \backslash \Sigma_{0}\right\} .
$$

Then we consider the quantity

$$
\triangle(K, R)=\frac{1}{2} \int_{B_{R}}\left|\nabla u_{\Sigma_{0}}\right|^{2}-\left|\nabla u_{K, R}\right|^{2} d x \geq 0 .
$$

Observe that, since $u_{K, R}$ is an admissible competitor in the definition of $\triangle\left(K, R^{\prime}\right)$ for $R^{\prime}>R$, then $R \mapsto \triangle(K, R)$ is nondecreasing. Therefore the following limit is well defined

$$
\triangle(K)=\lim _{R \rightarrow+\infty} \triangle(K, R) .
$$

Proposition 5.5. For all $K$ compact connected containing the origin one has

$$
\triangle(K)=-\mathcal{J}(K)
$$

Proof. Let $R^{\prime}>R>0$ be given. Extending $u_{K, R}$ by $u_{\Sigma_{0}}$ outside $B_{R}$, using that $w=u_{K, R}-u_{\Sigma_{0}} \in H^{1}\left(\mathbb{R}^{2} \backslash\left(\Sigma_{0} \cup K\right)\right)$ and noticing that

$$
\int_{\partial B_{R^{\prime}}} u_{\Sigma_{0}} \frac{\partial w}{\partial \nu} d x=0
$$

for all $R^{\prime}>R$, we deduce that

$$
\begin{aligned}
\mathcal{J}(K) & \leq \frac{1}{2} \int_{\mathbb{R}^{2}}|\nabla w|^{2} d x+\int_{B_{R^{\prime}}}\left\langle\nabla\left(u_{\Sigma_{0}}\right), \nabla w\right\rangle d x \\
& =\frac{1}{2} \int_{B_{R}}\left|\nabla u_{K, R}-\nabla u_{\Sigma_{0}}\right|^{2} d x+\int_{B_{R^{\prime}}}\left\langle\nabla\left(u_{\Sigma_{0}}\right), \nabla u_{K, R}\right\rangle d x-\int_{B_{R}}\left|\nabla u_{\Sigma_{0}}\right|^{2} d x \\
& =\frac{1}{2} \int_{B_{R}}\left|\nabla u_{K, R}\right|^{2} d x-\frac{1}{2} \int_{B_{R}}\left|\nabla u_{\Sigma_{0}}\right|^{2} d x \\
& =-\triangle(K, R) .
\end{aligned}
$$

Letting $R \rightarrow+\infty$ we get

$$
\mathcal{J}(K) \leq-\triangle(K)
$$

Next, let us denote by

$$
F_{R, K}(u):=\frac{1}{2} \int_{\mathbb{R}^{2}}|\nabla u|^{2} d x+\int_{B_{R}}\left\langle\nabla\left(u_{\Sigma_{0}}\right), \nabla u\right\rangle d x-\int_{\partial B_{R}} u_{\Sigma_{0}} \frac{\partial u}{\partial \nu} d \mathcal{H}^{1}
$$


so that

$$
\mathcal{J}(K)=\min _{u \in H^{1}\left(\mathbb{R}^{2} \backslash\left(\Sigma_{0} \cup K\right)\right)} F_{R, K}(u) .
$$

Let $\varepsilon, R>0$ be fixed and let $v_{\varepsilon} \in C^{\infty}\left(\mathbb{R}^{2} \backslash\left(\Sigma_{0} \cup K\right)\right)$ be a function with compact support in $\mathbb{R}^{2}$ such that

$$
F_{R, K}\left(v_{\varepsilon}\right) \leq \mathcal{J}(K)+\varepsilon .
$$

This is always possible because the space of functions in $C^{\infty}\left(\mathbb{R}^{2} \backslash\left(\Sigma_{0} \cup K\right)\right)$ with compact support is dense in $H^{1}\left(\mathbb{R}^{2} \backslash\left(\Sigma_{0} \cup K\right)\right)$ and $F_{R, K}(u)$ is continuous with respect to the topology of $H^{1}\left(\mathbb{R}^{2} \backslash\left(\Sigma_{0} \cup K\right)\right)$.

Let $R_{\varepsilon}>R$ be a big radius in such a way that $B_{R_{\varepsilon}}(0)$ contains the support of $v_{\varepsilon}$. Then, for any $R^{\prime}>R_{\varepsilon}$, the function $w=v_{\varepsilon}+u_{\Sigma_{0}}$ is an admissible competitor for the minimization which defines $\triangle\left(K, R^{\prime}\right)$. Thus

$$
\begin{aligned}
-\triangle\left(K, R^{\prime}\right) & \leq \frac{1}{2} \int_{B_{R^{\prime}}}|\nabla w|^{2}-\frac{1}{2} \int_{B_{R^{\prime}}}\left|\nabla u_{\Sigma_{0}}\right|^{2} \\
& =\frac{1}{2} \int_{B_{R^{\prime}}}\left|\nabla v_{\varepsilon}+\nabla u_{\Sigma_{0}}\right|^{2}-\frac{1}{2} \int_{B_{R^{\prime}}}\left|\nabla u_{\Sigma_{0}}\right|^{2} \\
& =\frac{1}{2} \int_{B_{R^{\prime}}}\left|\nabla v_{\varepsilon}\right|^{2}+\int_{B_{R^{\prime}}}\left\langle\nabla v_{\varepsilon}, \nabla u_{\Sigma_{0}}\right\rangle \\
& =\frac{1}{2} \int_{\mathbb{R}^{2}}\left|\nabla v_{\varepsilon}\right|^{2}+\int_{B_{R^{\prime}} \backslash B_{R}}\left\langle\nabla v_{\varepsilon}, \nabla u_{\Sigma_{0}}\right\rangle+\int_{B_{R}}\left\langle\nabla v_{\varepsilon}, \nabla u_{\Sigma_{0}}\right\rangle \\
& =\frac{1}{2} \int_{\mathbb{R}^{2}}\left|\nabla v_{\varepsilon}\right|^{2}-\int_{\partial B_{R}} u_{\Sigma_{0}} \frac{\partial v_{\varepsilon}}{\partial \nu}+\int_{B_{R}}\left\langle\nabla v_{\varepsilon}, \nabla u_{\Sigma_{0}}\right\rangle \\
& =F_{R, K}\left(v_{\varepsilon}\right) \leq \mathcal{J}(K)+\varepsilon
\end{aligned}
$$

and finally letting $R^{\prime} \rightarrow+\infty$ and then $\varepsilon \rightarrow 0$ we get

$$
-\triangle(K) \leq \mathcal{J}(K)
$$

which together with (5.13) yields (5.11).

Proposition 5.6. For all $K$ compact connected containing the origin we have

$$
\begin{aligned}
\triangle(K, R) & =\frac{1}{\lambda} \triangle(\lambda K, \lambda R) \\
\triangle(K) & =\lim _{\lambda \rightarrow 0} \frac{1}{\lambda} \triangle(\lambda K, R) \quad \forall R .
\end{aligned}
$$

Proof. The proof of (5.14) follows easily by the change of variable $y=\lambda x$. Indeed, if $w(x)$ is the minimizer of (5.10) for some $R$, then $\sqrt{\lambda} w(x / \lambda)$ is the minimizer of 
the same problem associated to $\lambda K$ in $B_{\lambda R}$ (because $u_{\Sigma_{0}}$ is $1 / 2$-homogeneous thus $\sqrt{\lambda} w(x / \lambda)=u_{\Sigma_{0}}$ on $\partial B_{\lambda R}$ when $w=u_{\Sigma_{0}}$ on $\left.B_{R}\right)$. In other words,

$$
u_{\lambda K, \lambda R}(x)=\sqrt{\lambda} u_{K, R}(x / \lambda) .
$$

It follows that

$$
\begin{aligned}
\triangle(\lambda K, \lambda R) & =\int_{B_{\lambda R}}\left|\nabla u_{\Sigma_{0}}\right|^{2}-\int_{B_{\lambda R}}\left|\nabla u_{\lambda K, \lambda R}\right|^{2} \\
& =\lambda \int_{B_{R}}\left|\nabla u_{\Sigma_{0}}\right|^{2}-\lambda \int_{B_{R}}\left|\nabla u_{K, R}\right|^{2} \\
& =\lambda \triangle(K, R),
\end{aligned}
$$

which proves (5.14). But then we directly get

$$
\triangle\left(K, \frac{1}{\lambda} R\right)=\frac{1}{\lambda} \triangle(\lambda K, R)
$$

which, by taking $\lambda \rightarrow 0$, implies (5.15).

We are now ready to give a proof for Proposition (5.4).

Proof of Proposition 5.4. It is equivalent to prove that

$$
\max _{K ; \mathcal{H}^{1}\left(K \backslash \Sigma_{0}\right) \leq \ell} \triangle(K)=\triangle([0, \ell] \times\{0\}) .
$$

Actually, it is enough to prove that

$$
\triangle(K) \leq \mathcal{H}^{1}\left(K \backslash \Sigma_{0}\right),
$$

because some elementary computations yield (see [Dav05b, Page 406])

$$
\triangle([0, \ell] \times\{0\})=\ell .
$$

Now to prove (5.16), we argue by contradiction. If it is not true then one can find a set $K$ such that

$$
\triangle(K)>\mathcal{H}^{1}\left(K \backslash \Sigma_{0}\right) .
$$

Thanks to (5.15) we can find $R>0$ such that

$$
\frac{1}{\lambda} \triangle(\lambda K, R)>\mathcal{H}^{1}\left(K \backslash \Sigma_{0}\right) .
$$

If $K^{\prime}:=\lambda K$ we have found a set such that

$$
\triangle\left(K^{\prime}, R\right)>\mathcal{H}^{1}\left(K^{\prime} \backslash \Sigma_{0}\right)
$$

But then we have that

$$
\int_{B_{R}}\left|\nabla u_{K^{\prime}, R}\right|^{2}+\mathcal{H}^{1}\left(\Sigma_{0} \cup K^{\prime} \cap B_{R}\right)<\int_{B_{R}}\left|\nabla u_{\Sigma_{0}}\right|^{2}+\mathcal{H}^{1}\left(\Sigma_{0} \cap B_{R^{\prime}}\right)
$$

which contradicts the fact that $\left(u_{\Sigma_{0}}, \Sigma_{0}\right)$ is a global minimizer [BD01]. 


\subsection{A compliance problem}

If $\Omega \subset \mathbb{R}^{2}$ and $f \in L^{\infty}(\Omega)$, the so called compliance energy associated to a membrane attached to $\partial \Omega$ and subject to a vertical force $f$ is $\frac{1}{2} \int_{\Omega} u f d x$, where $u \in H_{0}^{1}(\Omega)$ is the unique solution for the problem $-\Delta u=f$ in $\Omega$.

In [BS07], the following free discontinuity problem is introduced, also studied in [Til12, CLLS]. It is commonly called the "Glue-problem" by Buttazzo, and has apparently nothing to do with the Mumford-Shah problem. The purpose of this section is to show that in some sense it is the dual problem of a Mumford-Shah type problem.

For any $\Sigma \subset \bar{\Omega}$ compact and connected, let us denote by $u_{\Sigma}$ the unique solution for the problem

$$
\min _{u \in H_{0}^{1}(\Omega \backslash \Sigma)}\left(\frac{1}{2} \int_{\Omega}|\nabla u|^{2} d x-\int_{\Omega} u f d x\right) .
$$

The function $u_{\Sigma}$ is then a weak solution for the problem

$$
\left\{\begin{array}{c}
-\Delta u=f \quad \text { in } \Omega \backslash \Sigma \\
u=0 \quad \text { on } \partial \Omega \cup \Sigma
\end{array}\right.
$$

We denote by $C(\Sigma)$ the compliance energy associated to $\Sigma$ and defined by

$$
C(\Sigma)=\frac{1}{2} \int_{\Omega}\left|\nabla u_{\Sigma}\right|^{2} d x=\frac{1}{2} \int_{\Omega} u_{\Sigma} f d x .
$$

Then $\lambda>0$ being a fixed parameter we minimize

$$
\min _{\Sigma}\left(C(\Sigma)+\lambda \mathcal{H}^{1}(\Sigma)\right)
$$

where the minimum is taken among all compact connected sets $\Sigma \subset \bar{\Omega}$.

The interpretation of the shape optimisation problem (5.18) is the following: the set $\Sigma$ is supposed to be the location of some glue-line that attaches a membrane on $\Omega$ (as well as $\partial \Omega$ ). Then the minimizer $\Sigma$ tries to find the best location for the glue in order to minimize the compliance energy of a corresponding membrane, subject to the force $f$, while the penalization by $\lambda \mathcal{H}^{1}$ takes into account, for instance, the cost of the glue.

Remarkably, the above problem can be seen as a dual problem for a certain problem of Mumford-Shah type. Let us do it formally. For any given $u \in H_{0}^{1}(\Omega \backslash \Sigma)$ and $\Phi \in L^{2}\left(\Omega, \mathbb{R}^{2}\right)$, we can write

$$
\begin{aligned}
\int_{\Omega}\left|\nabla u^{2}\right| & =\int_{\Omega}|\nabla u-\Phi|^{2}+2 \int_{\Omega}\langle\nabla u, \Phi\rangle-\int_{\Omega}|\Phi|^{2} \\
& \geq 2 \int_{\Omega}\langle\nabla u, \Phi\rangle-\int_{\Omega}|\Phi|^{2}
\end{aligned}
$$


and since we have equality for $\Phi=\nabla u$ we then obtain the famous Legendre transformation

$$
\int_{\Omega}\left|\nabla u^{2}\right|=\sup _{\Phi \in L^{2}}\left(2 \int_{\Omega}\langle\nabla u, \Phi\rangle-\int_{\Omega}|\Phi|^{2}\right) .
$$

Therefore, the problem in (5.17) can be written as

$$
\inf _{u \in H_{0}^{1}(\Omega \backslash \Sigma)}\left(\sup _{\Phi \in L^{2}}\left(\int_{\Omega}\langle\nabla u, \Phi\rangle-\frac{1}{2} \int_{\Omega}|\Phi|^{2}\right)-\int_{\Omega} u f d x\right)
$$

Now we assume formally that the inf and sup can be exchanged, which leads to

$$
\begin{aligned}
& \sup _{\Phi \in L^{2}} \inf _{u \in H_{0}^{1}(\Omega \backslash \Sigma)}\left(\int_{\Omega}\langle\nabla u, \Phi\rangle-\frac{1}{2} \int_{\Omega}|\Phi|^{2}-\int_{\Omega} u f d x\right) \\
= & \sup _{\Phi \in L^{2}} \inf _{u \in H_{0}^{1}(\Omega \backslash \Sigma)}\left(\int_{\Omega} u(\operatorname{div} \Phi-f)-\frac{1}{2} \int_{\Omega}|\Phi|^{2}\right),
\end{aligned}
$$

where we used an integration by parts in $\Omega \backslash \Sigma$ and the fact that $u=0$ on $\partial \Omega \cup \Sigma$ to get the last equality. The infimum in the above is $-\infty$ except if $\operatorname{div} \Phi=f$ in $\Omega \backslash \Sigma$. In conclusion we have obtained that

$$
\min _{u \in H_{0}^{1}(\Omega \backslash \Sigma)}\left(\frac{1}{2} \int_{\Omega}|\nabla u|^{2} d x-\int_{\Omega} u f d x\right)=-\min \left\{\frac{1}{2} \int_{\Omega}|\Phi|^{2} d x ; \operatorname{div} \Phi=f \text { in } \Omega \backslash \Sigma\right\} .
$$

If we set

$$
\mathcal{D}:=\left\{\Phi \in L^{2}(\Omega) ; \operatorname{div} \Phi=f \text { in } \Omega \backslash \Sigma\right\}
$$

and

$$
\mathcal{B}:=\{(\Phi, \Sigma) ; \Sigma \text { compact connected and } \Phi \in \mathcal{D}\},
$$

then the compliance problem (5.18) is reduced to

$$
\min _{(\Phi, \Sigma) \in \mathcal{B}} \int_{\Omega}|\Phi|^{2} d x+\lambda \mathcal{H}^{1}(\Sigma)
$$

Up to now, everything can be rigorously justified (see [CLLS, BLS15]). But to go further, we will be more optimistic, and try to write $\Phi$ as the gradient of something. To do so, we first solve the Dirichlet problem

$$
\left\{\begin{array}{c}
-\Delta g=f \quad \text { in } \Omega \\
g=0 \text { on } \partial \Omega
\end{array}\right.
$$

and call $g$ its solution, which depends on $\Omega$ and $f$. Then the vector field $\Phi+\nabla g$ has zero divergence in $\Omega \backslash \Sigma$. It would be very convenient to apply a theorem of De Rham type in order to deduce that

$$
\Phi+\nabla g=\nabla \Psi^{T}
$$


for some vector field $\Psi$. Unfortunately, this conclusion only holds upon very restrictive topological condition on the domain $\Omega \backslash \Sigma$ (for instance simply connected), which has no chances to hold in general under our setting. But we can believe that it holds locally, or we could also add it as a new constraint in our problem, and this would lead to consider the following Mumford-Shah type functional,

$$
\min _{(u, \Sigma)} \int_{\Omega}|\nabla u-G|^{2}+\lambda \mathcal{H}^{1}(\Sigma)
$$

where $G=\nabla g^{T}$ depends on the datum of the problem ( $\Omega$ and $f$ ), and where the minimum is taken among all $\Sigma \subset \Omega$ compact connected and $u$ belonging to $W^{1,2}(\Omega \backslash \Sigma)$.

In [CLLS] it is proved that the optimal set $\Sigma$ is a finite union of $C^{1}$ curves meeting only by three, using this duality approach.

\section{References}

[ABDM03] Giovanni Alberti, Guy Bouchitté, and Gianni Dal Maso. The calibration method for the Mumford-Shah functional and free-discontinuity problems. Calc. Var. Partial Differential Equations, 16(3):299-333, 2003.

[ACF84] Hans Wilhelm Alt, Luis A. Caffarelli, and Avner Friedman. Variational problems with two phases and their free boundaries. Trans. Amer. Math. Soc., 282(2):431-461, 1984.

[AFH03] Luigi Ambrosio, Nicola Fusco, and John E. Hutchinson. Higher integrability of the gradient and dimension of the singular set for minimisers of the Mumford-Shah functional. Calc. Var. Partial Differential Equations, 16(2):187-215, 2003.

[AFP97] Luigi Ambrosio, Nicola Fusco, and Diego Pallara. Partial regularity of free discontinuity sets. II. Ann. Scuola Norm. Sup. Pisa Cl. Sci. (4), 24(1):39-62, 1997.

[AFP00] Luigi Ambrosio, Nicola Fusco, and Diego Pallara. Functions of bounded variation and free discontinuity problems. Oxford Mathematical Monographs. The Clarendon Press Oxford University Press, New York, 2000.

[Amb89] L. Ambrosio. A compactness theorem for a new class of functions of bounded variation. Boll. Un. Mat. Ital. B (7), 3(4):857-881, 1989. 
[AP97] Luigi Ambrosio and Diego Pallara. Partial regularity of free discontinuity sets. I. Ann. Scuola Norm. Sup. Pisa Cl. Sci. (4), 24(1):1-38, 1997.

[AT92] L. Ambrosio and V. M. Tortorelli. On the approximation of free discontinuity problems. Boll. Un. Mat. Ital. B (7), 6(1):105-123, 1992.

[BC94] Giovanni Bellettini and Alessandra Coscia. Discrete approximation of a free discontinuity problem. Numer. Funct. Anal. Optim., 15(3-4):201$224,1994$.

[BC00] Blaise Bourdin and Antonin Chambolle. Implementation of an adaptive finite-element approximation of the Mumford-Shah functional. Numer. Math., 85(4):609-646, 2000.

[BCL15] Jean-François Babadjian, Antonin Chambolle, and Antoine Lemenant. Energy release rate for non-smooth cracks in planar elasticity. J. Éc. polytech. Math., 2:117-152, 2015.

[BD01] Alexis Bonnet and Guy David. Cracktip is a global Mumford-Shah minimizer. Astérisque, (274):vi+259, 2001.

[BG14] Jean-François Babadjian and Alessandro Giacomini. Existence of strong solutions for quasi-static evolution in brittle fracture. Ann. Sc. Norm. Super. Pisa Cl. Sci. (5), 13(4):925-974, 2014.

[BL14] Dorin Bucur and Stephan Luckhaus. Monotonicity formula and regularity for general free discontinuity problems. Arch. Ration. Mech. Anal., 211(2):489-511, 2014.

[BLS15] Matthieu Bonnivard, Antoine Lemenant, and Filippo Santambrogio. Approximation of length minimization problems among compact connected sets. SIAM J. Math. Anal., 47(2):1489-1529, 2015.

[Bon96] A. Bonnet. On the regularity of edges in image segmentation. Ann. Inst. H. Poincaré Anal. Non Linéaire, 13(4):485-528, 1996.

[Bou99] Blaise Bourdin. Image segmentation with a finite element method. M2AN Math. Model. Numer. Anal., 33(2):229-244, 1999.

[BS07] Giuseppe Buttazzo and Filippo Santambrogio. Asymptotical compliance optimization for connected networks. Netw. Heterog. Media, 2(4):761-777, 2007. 
[CDM99] Antonin Chambolle and Gianni Dal Maso. Discrete approximation of the Mumford-Shah functional in dimension two. M2AN Math. Model. Numer. Anal., 33(4):651-672, 1999.

[CFM09] A. Chambolle, G. A. Francfort, and J.-J. Marigo. When and how do cracks propagate? J. Mech. Phys. Solids, 57(9):1614-1622, 2009.

[CFM10] Antonin Chambolle, Gilles A. Francfort, and Jean-Jacques Marigo. Revisiting energy release rates in brittle fracture. J. Nonlinear Sci., 20(4):395-424, 2010.

[CGP08] Antonin Chambolle, Alessandro Giacomini, and Marcello Ponsiglione. Crack initiation in brittle materials. Arch. Ration. Mech. Anal., 188(2):309-349, 2008.

[Cha03] Antonin Chambolle. A density result in two-dimensional linearized elasticity, and applications. Arch. Ration. Mech. Anal., 167(3):211233, 2003.

[CL90] M. Carriero and A. Leaci. Existence theorem for a Dirichlet problem with free discontinuity set. Nonlinear Anal., 15(7):661-677, 1990.

[CL13] Antonin Chambolle and Antoine Lemenant. The stress intensity factor for non-smooth fractures in antiplane elasticity. Calc. Var. Partial Differential Equations, 47(3-4):589-610, 2013.

[CLLS] Antonin Chambolle, Jimmy Lamboley, Antoine Lemenant, and Eugene Stepanov. Regularity for optimal compliance problem with length penalization. preprint Arxiv 2015.

[Dau92] Monique Dauge. Neumann and mixed problems on curvilinear polyhedra. Integral Equations Operator Theory, 15(2):227-261, 1992.

[Dav96] Guy David. $C^{1}$-arcs for minimizers of the Mumford-Shah functional. SIAM J. Appl. Math., 56(3):783-888, 1996.

[Dav05a] Guy David. Open questions on the Mumford-Shah functional. In Perspectives in analysis, volume 27 of Math. Phys. Stud., pages 37-49. Springer, Berlin, 2005.

[Dav05b] Guy David. Singular sets of minimizers for the Mumford-Shah functional, volume 233 of Progress in Mathematics. Birkhäuser Verlag, Basel, 2005. 
[Dav09] Guy David. Hölder regularity of two-dimensional almost-minimal sets in $\mathbb{R}^{n}$. Ann. Fac. Sci. Toulouse Math. (6), 18(1):65-246, 2009.

[Dav10] Guy David. $C^{1+\alpha}$-regularity for two-dimensional almost-minimal sets in $\mathbb{R}^{n}$. J. Geom. Anal., 20(4):837-954, 2010.

[DDPT08] Guy David, Thierry De Pauw, and Tatiana Toro. A generalization of Reifenberg's theorem in $\mathbb{R}^{3}$. Geom. Funct. Anal., 18(4):1168-1235, 2008.

[DGCL89] E. De Giorgi, M. Carriero, and A. Leaci. Existence theorem for a minimum problem with free discontinuity set. Arch. Rational Mech. Anal., 108(3):195-218, 1989.

[DK91] Françoise Dibos and Georges Koepfler. Propriété de régularité des contours d'une image segmentée. C. R. Acad. Sci. Paris Sér. I Math., 313(9):573-578, 1991.

[DL02] Guy David and Jean-Christophe Léger. Monotonicity and separation for the Mumford-Shah problem. Ann. Inst. H. Poincaré Anal. Non Linéaire, 19(5):631-682, 2002.

[DLF13] C. De Lellis and M. Focardi. Higher integrability of the gradient for minimizers of the $2 d$ Mumford-Shah energy. J. Math. Pures Appl. (9), 100(3):391-409, 2013.

[DMFT05] Gianni Dal Maso, Gilles A. Francfort, and Rodica Toader. Quasistatic crack growth in nonlinear elasticity. Arch. Ration. Mech. Anal., 176(2):165-225, 2005.

[DMMM00] Gianni Dal Maso, Maria Giovanna Mora, and Massimiliano Morini. Local calibrations for minimizers of the Mumford-Shah functional with rectilinear discontinuity sets. J. Math. Pures Appl. (9), 79(2):141-162, 2000 .

[DMMS92] G. Dal Maso, J.-M. Morel, and S. Solimini. A variational method in image segmentation: existence and approximation results. Acta Math., 168(1-2):89-151, 1992.

[DMT02] Gianni Dal Maso and Rodica Toader. A model for the quasi-static growth of brittle fractures: existence and approximation results. Arch. Ration. Mech. Anal., 162(2):101-135, 2002. 
[DPF14] Guido De Philippis and Alessio Figalli. Higher integrability for minimizers of the Mumford-Shah functional. Arch. Ration. Mech. Anal., 213(2):491-502, 2014.

[DPS99] Thierry De Pauw and Didier Smets. On explicit solutions for the problem of Mumford and Shah. Commun. Contemp. Math., 1(2):201-212, 1999.

[DS91] G. David and S. Semmes. Singular integrals and rectifiable sets in $\mathbf{R}^{n}$ : Beyond Lipschitz graphs. Astérisque, (193):152, 1991.

[DS96a] G. David and S. Semmes. On the singular sets of minimizers of the Mumford-Shah functional. J. Math. Pures Appl. (9), 75(4):299-342, 1996.

[DS96b] Guy David and Stephen Semmes. Uniform rectifiability and singular sets. Ann. Inst. H. Poincaré Anal. Non Linéaire, 13(4):383-443, 1996.

[FL03] Gilles A. Francfort and Christopher J. Larsen. Existence and convergence for quasi-static evolution in brittle fracture. Comm. Pure Appl. Math., 56(10):1465-1500, 2003.

[FM98] G. A. Francfort and J.-J. Marigo. Revisiting brittle fracture as an energy minimization problem. J. Mech. Phys. Solids, 46(8):1319-1342, 1998.

[Foc] Matteo Focardi.

[Fus03] Nicola Fusco. An overview of the Mumford-Shah problem. Milan J. Math., 71:95-119, 2003.

[Iur13] Flaviana Iurlano. Fracture and plastic models as $\Gamma$-limits of damage models under different regimes. Adv. Calc. Var., 6(2):165-189, 2013.

[KKT10] A. M. Khludnev, V. A. Kovtunenko, and A. Tani. On the topological derivative due to kink of a crack with non-penetration. Anti-plane model. J. Math. Pures Appl. (9), 94(6):571-596, 2010.

[KLM05] Herbert Koch, Giovanni Leoni, and Massimiliano Morini. On optimal regularity of free boundary problems and a conjecture of De Giorgi. Comm. Pure Appl. Math., 58(8):1051-1076, 2005.

[Lég94] J.-C. Léger. Une remarque sur la régularité d'une image segmentée. J. Math. Pures Appl. (9), 73(6):567-577, 1994. 
[Leg99] J. C. Leger. Flatness and finiteness in the Mumford-Shah problem. J. Math. Pures Appl. (9), 78(4):431-459, 1999.

[Lem08] Antoine Lemenant. Sur la régularité des minimiseurs de Mumford-Shah en dimension 3 et supérieure. Thesis Université Paris Sud XI, Orsay, 2008.

[Lem09] Antoine Lemenant. On the homogeneity of global minimizers for the Mumford-Shah functional when $K$ is a smooth cone. Rend. Semin. Mat. Univ. Padova, 122:129-159, 2009.

[Lem10] Antoine Lemenant. Energy improvement for energy minimizing functions in the complement of generalized Reifenberg-flat sets. Ann. Sc. Norm. Super. Pisa Cl. Sci. (5), 9(2):351-384, 2010.

[Lem11] Antoine Lemenant. Regularity of the singular set for Mumford-Shah minimizers in $\mathbb{R}^{3}$ near a minimal cone. Ann. Sc. Norm. Super. Pisa Cl. Sci. (5), 10(3):561-609, 2011.

[Lem12] Antoine Lemenant. Un théorème de régularité pour les minimiseurs de Mumford-Shah dans $\mathbb{R}^{3}$. In Seminaire: Equations aux Dérivées Partielles. 2009-2010, Sémin. Équ. Dériv. Partielles, pages Exp. No. XXV, 11. École Polytech., Palaiseau, 2012.

[Lem14] Antoine Lemenant. A rigidity result for global mumford-shah minimizers in dimension three. J. Math. Pures. App., 2014.

[LF13] Camillo De Lellis and Matteo Focardi. Density lower bound estimates for local minimizers of the 2d Mumford-Shah energy. Manuscripta Math., 142(1-2):215-232, 2013.

[LFR13] Camillo De Lellis, Matteo Focardi, and Berardo Ruffini. A note on the hausdorff dimension of the singular set for minimizers of the mumfordshah energy. Adv. Calc. var., 2013.

[LT11] Giuliano Lazzaroni and Rodica Toader. Energy release rate and stress intensity factor in antiplane elasticity. J. Math. Pures Appl. (9), 95(6):565-584, 2011.

[Maz09] Vladimir Maz'ya. On the boundedness of first derivatives for solutions to the Neumann-Laplace problem in a convex domain. J. Math. Sci. (N. Y.), 159(1):104-112, 2009. Problems in mathematical analysis. No. 40. 
[Mer07] Benoît Merlet. Numerical study of a new global minimizer for the Mumford-Shah functional in $\mathbf{R}^{3}$. ESAIM Control Optim. Calc. Var., 13(3):553-569, 2007.

[MM01] Maria Giovanna Mora and Massimiliano Morini. Local calibrations for minimizers of the Mumford-Shah functional with a regular discontinuity set. Ann. Inst. H. Poincaré Anal. Non Linéaire, 18(4):403-436, 2001.

[Mor97] Jean-Michel Morel. The Mumford-Shah conjecture in image processing. Astérisque, (241):Exp. No. 813, 4, 221-242, 1997. Séminaire Bourbaki, Vol. 1995/96.

[Mor02a] Maria Giovanna Mora. The calibration method for free-discontinuity problems on vector-valued maps. J. Convex Anal., 9(1):1-29, 2002.

[Mor02b] Maria Giovanna Mora. Local calibrations for minimizers of the Mumford-Shah functional with a triple junction. Commun. Contemp. Math., 4(2):297-326, 2002.

[Mor02c] Massimiliano Morini. Global calibrations for the non-homogeneous Mumford-Shah functional. Ann. Sc. Norm. Super. Pisa Cl. Sci. (5), $1(3): 603-648,2002$.

[MS89] David Mumford and Jayant Shah. Optimal approximations by piecewise smooth functions and associated variational problems. Comm. Pure Appl. Math., 42(5):577-685, 1989.

[MS95] Jean-Michel Morel and Sergio Solimini. Variational methods in image segmentation. Progress in Nonlinear Differential Equations and their Applications, 14. Birkhäuser Boston, Inc., Boston, MA, 1995. With seven image processing experiments.

[MS01] Francesco Maddalena and Sergio Solimini. Regularity properties of free discontinuity sets. Ann. Inst. H. Poincaré Anal. Non Linéaire, 18(6):675-685, 2001.

[Rig00] Séverine Rigot. Big pieces of $C^{1, \alpha}$-graphs for minimizers of the Mumford-Shah functional. Ann. Scuola Norm. Sup. Pisa Cl. Sci. (4), 29(2):329-349, 2000.

[Sia03] Anthony Siaudeau. Ahlfors-régularité des quasi-minima de MumfordShah. J. Math. Pures Appl. (9), 82(12):1697-1731, 2003.

[Sol97] Sergio Solimini. Simplified excision techniques for free disontinuity problems in several variables. J. Funct. Anal., 151(1):1-34, 1997. 
[Tay76] Jean E. Taylor. The structure of singularities in soap-bubble-like and soap-film-like minimal surfaces. Ann. of Math. (2), 103(3):489-539, 1976.

[Til12] Paolo Tilli. Compliance estimates for two-dimensional problems with Dirichlet region of prescribed length. Netw. Heterog. Media, 7(1):127136, 2012. 